\title{
Nonsingular Integral Sliding Mode Attitude Control for Rigid-Flexible Coupled Spacecraft with High-Inertia Rotating Appendages
}

\author{
Gaowang Zhang, Xueqin Chen $\mathbb{D}$, Ruichen Xi, and Huayi Li \\ Research Center of the Satellite Technology, Harbin Institute of Technology, Harbin 150080, China \\ Correspondence should be addressed to Xueqin Chen; cxqhit@163.com
}

Received 21 September 2020; Revised 2 January 2021; Accepted 18 January 2021; Published 16 February 2021

Academic Editor: Rui Wang

Copyright (c) 2021 Gaowang Zhang et al. This is an open access article distributed under the Creative Commons Attribution License, which permits unrestricted use, distribution, and reproduction in any medium, provided the original work is properly cited.

\begin{abstract}
This study addresses the challenge of attitude tracking control for a rigid-flexible spacecraft with high-inertia rotating appendages. The Lagrange method was used to establish the kinematic and dynamic models of the spacecraft. The translation and rotation of the spacecraft, vibrations of solar panels, and imbalance caused by the rotating appendages, which cause a complex control problem, were considered. To address the complex control problem, a novel, fast nonsingular integral sliding mode control method is proposed to perform the attitude tracking function of spacecraft. A sliding mode control law was established for the high-inertia appendages to maintain an appropriate angular velocity during rotation. Finally, the effectiveness of the proposed attitude control law was verified by numerical simulations for a spacecraft with high-inertia rotating appendages and symmetrical flexible solar panels.
\end{abstract}

\section{Introduction}

With the increased complexity of modern space missions, modern spacecraft carry many rigid and flexible components, such as manipulators, solar panels, antennas, and cameras. Additionally, many modern spacecraft components possess highly nonlinear characteristics. Owing to the strong nonlinearity of spacecraft systems and the improvements in the spacecraft attitude control and tracking accuracy, attitude control and tracking for spacecraft with multiple appendages have become a very complex problem.

Modern spacecraft are predominantly powered by large, lightweight solar panels. To absorb as much solar energy as possible, it is necessary that the solar panels rotate while the spacecraft moves to ensure the panels are oriented perpendicular to the incident light. Notably, during rapid maneuvering, the rotation and vibration of flexible components reduce the stiffness and increase the nonlinearity of the spacecraft system. These components are always coupled with the attitude and orbital motion of the rigid body of spacecraft, thus increasing the complexity of spacecraft attitude control and tracking problem $[1,2]$. Li et al. [3] used cooperative tracking protocols to address unknown nonlinear dynamics problems with an adaptive learning law, which solved the leader-following tracking problem of fractional-order multiagent systems. For multiagent systems with unknown disturbances and input saturation, Zhou et al. [4] proposed an event-triggered control method based on disturbance observer to solve the following consistency problem. Li et al. [5] proposed a finite-time fuzzy adaptive control (AC) scheme to address the finite-time adaptive fuzzy control problem for a class of multiinput multioutput (MIMO) nonlinear nonstrict feedback systems. Tong et al. [6] proposed a novel observerbased adaptive fuzzy output-feedback control method to solve the output-feedback backstepping control design problem in uncertain strict-feedback nonlinear systems. To solve the fault observer design problem of Markovian jumped systems, Chen et al. [7] proposed two types of adaptive observer methods that could avoid the sliding 
surface switching problem. Zhao et al. [8] proposed a control strategy combined with filtered backstepping and adaptive technique to solve the problem of adaptive finitetime attitude control for multiple spacecraft with unknown external disturbances. The solar panel of the Feng Yun-3 (FY-3) spacecraft rotates relative to the spacecraft body to ensure the solar panel generates sufficient energy for the system [9]. In addition, external disturbances, inertial variability, and nonlinearity pose difficulties for the design of fast and accurate attitude control schemes, such as proportional integral derivative (PID) control, active disturbance rejection control (ADRC) [10], AC [11, 12], sliding mode control (SMC) [13], and finite-time control (FTC) [14]. SMC exhibits excellent performance in uncertain nonlinear systems. Thus, various sliding mode variable structure control methods have been proposed for spacecraft attitude tracking, such as integral SMC [15], second-order SMC [16], and terminal SMC [17].

As a form of finite-time control, terminal SMC has attracted increasing attention owing to its robustness and anti-interference properties. Terminal SMC has been applied to spacecraft attitude control but with limitations because of singularity and slow convergence. Zhao [18] proposed a finite-time command filter backstepping approach based on a finite-time adaptive fuzzy tracking control scheme for a class of unknown nonlinear systems. In recent years, nonsingular terminal SMC has attracted increasing attention for applications in complex spacecraft attitude control owing to its advantageous characteristics. Lu et al. [19] proposed an effective SMC strategy for a nonlinear spacecraft model with external disturbances and inertial uncertainty that could effectively solve the problem of spacecraft attitude tracking control. Yousefpour et al. [20] proposed an integral terminal SMC method based on a disturbance observer for the tracking control of a hyperchaotic memory resistance oscillator. To solve the precise trajectory tracking control problem of unmanned vehicles, Lv et al. [21] proposed a nonsingular integral SMC law based on a finite-time disturbance observer. In [19-21], effective disturbance observers were designed for controlled systems to achieve high performance in the presence of inertia uncertainties and external disturbances. $\mathrm{Na}[22]$ proposed an alternative AC with prescribed performance to solve the output tracking problem of nonlinear systems with unknown dead zones. Chen et al. [23] designed an adaptive finite-time controller based on the fast TSM control principle to overcome the singularity problem of nonlinear systems. $\mathrm{Na}$ [22] and Chen et al. [23] designed effective attitude control schemes for systems with problems such as uncertain inertia terms, unknown disturbances, and sudden actuator failure. To solve the attitude tracking problem for a rigid platform spacecraft with rotating flexible appendages, Shi et al. [24] proposed the nonsingular terminal SMC algorithm for the finite-time tracking control of $n$-order nonlinear dynamical systems. To achieve underactuated attitude stabilization, Yue et al. [25] designed a higher-level SMC part to stabilize the angular velocity combined with a tracking control part to track desired angular velocities. Furthermore, [24, 25] demonstrated effective control laws for nonlinear dynamical systems to achieve finite-time convergence and strong robustness. Alshamali et al. [26] designed an observer-based backstepping controller to solve attitude tracking problems with the angles of satellite dynamics under external disturbances. Wang et al. [27] proposed an adaptive SMC scheme to control the chaotic oscillation of a complex seven-dimensional power system. The proposed scheme avoided the singularity problem and improved the accuracy of attitude control as well. Finally, Peng et al. [28] proposed a fast, nonsingular integral terminal SMC scheme for a class of nonlinear systems by introducing a power integral term. This type of scheme can be used to avoid the singularity problem without constraints and to achieve finite-time convergence of the system state. These control methods exhibit good performance and robustness in many systems but are unsuitable for rigid-flexible coupled spacecraft with high-inertia rotating appendages.

In recent years, the number of high-inertia rotating appendages in spacecraft has increased. "high inertia" is mainly in the relative sense, and high-inertia components generally refer to appendages with similar mass as spacecraft or high-inertia matrixes. However, no unified and quantifiable standard is available in the academic field. These components often contain both static and dynamic imbalances, thus producing an unbalanced disturbance torque. Therefore, higher accuracy is required for the attitude control of spacecraft. Miao et al. [29] designed a fast, nonsingular SMC method integrated with an adaptive method, which has been proven to have a higher convergence speed and increased robustness than previous terminal SMC methods. However, high-inertia components and flexible solar panels were not considered in their study. Gui et al. [30] proposed a novel integral TSM controller integrated with an adaptive fault-tolerant control law to solve the attitude tracking problem for a rigid spacecraft; however, a rigid-flexible coupled spacecraft was not considered. This TSM controller exhibited better control performance, including higher convergence speed and estimation accuracy, than the conventional controllers reported in [29, 30]. Xie [31] designed a composite compensation controller to account for the control torques and reduce the impulse characteristics of the disturbance torque during the acceleration of rotating components. Further, the effectiveness of the controller was proven using a semiphysical simulation system; additionally, the coupling effect between the high-inertia components and platform and the characteristics of unbalanced disturbance torque were analyzed. In addition, a PD control algorithm combined with repetitive control was designed. Regarding the unbalanced characteristics of high-inertia rotating appendages, Chai $[32,33]$ used the Kalman filter method to effectively and accurately estimate unbalanced interference torques caused by appendage rotation.

Among the broad array of studies conducted to date, only a few studies address the problem of attitude tracking control of rigid-flexible coupled platforms with high-inertia components. To bridge this divide, this study proposes a novel, fast integral SMC strategy for spacecraft with high-inertia components and flexible solar panels and demonstrates the applicability of this approach to facilitate the attitude tracking of 
spacecraft systems. The primary contributions of this work are listed below:

(1) A rigid-flexible coupling spacecraft with rotating components is considered, and the mathematical and dynamic models are established

(2) An attitude control law for the high-inertia components of a spacecraft system, integrated with a novel, fast nonsingular integral SMC law for symmetrical solar panels, and a spacecraft platform to enable accurate spacecraft attitude tracking are demonstrated

The remainder of this paper is organized as follows. Section 2 introduces the dynamic and kinematic models for spacecraft attitude tracking with high-inertia rotating appendages and symmetrical solar panels. Section 3 introduces the proposed fast nonsingular integral SMC law. Section 4 presents the numerical simulation results to demonstrate the effectiveness of the control algorithm. Finally, Section 5 concludes the study.

\section{Preliminary Modeling}

A rigid spacecraft model with large, rigid rotating inertial components and symmetrical flexible solar panels was considered in this study. The coordinate system included the inertial reference frame $O X Y Z$, orbital reference frame $O X_{o} Y_{o} Z_{o}$, spacecraft platform reference frame $o x_{s} y_{s} z_{s}$, large rigid rotating component body-fixed frame $o x_{p} y_{p} z_{p}$, and flexible solar panels body-fixed frame $o x_{a} y_{a} z_{a}$ (Figure 1)-these were defined as $R, R_{o}, R_{s}, R_{p}$, and $R_{a}$, respectively. The $y$-axes of $R_{s}, R_{p}$, and $R_{a}$ were parallel. The flexible solar panels were installed on the $\pm y$-axis, and the large rigid rotating components were installed on the $x$-axis of the platform coordinate system $R$.

The rigid-flexible coupling spacecraft model consisted of kinematic and dynamic equations, which are described in the following sections.

2.1. Kinematic Model of the Spacecraft System. Because the Euler angle has a singularity, the Euler equation is not suitable for spacecraft with a wide range of attitude motion. To avoid the singularity and complex calculations, the unit quaternions are often used to describe the kinematics of a spacecraft. A unit quaternion is expressed as $Q_{s}=\left[\begin{array}{ll}q_{0} & \mathbf{q}_{s}^{T}\end{array}\right]^{T}=\left[\begin{array}{llll}q_{0} & q_{1} & q_{2} & q_{3}\end{array}\right]^{T}$, where $q_{0}$ and $\mathbf{q}_{s}=\left[\begin{array}{lll}q_{1} & q_{2} & q_{3}\end{array}\right]^{T}$ are the scalar and vector parts of the quaternion, respectively, and $q_{0}$ and $\boldsymbol{q}_{\boldsymbol{s}}$ satisfy the condition $q_{0}^{2}+\mathbf{q}_{s}^{T} \mathbf{q}_{s}=1$.

The kinematic equation with the quaternion of spacecraft can be given as

$$
\dot{\mathbf{Q}}=\frac{1}{2} E\left(\mathbf{q}_{s}\right) * \omega_{b}=\frac{1}{2}\left[\begin{array}{c}
-\mathbf{q}_{s}^{T} \\
\widetilde{\mathbf{q}}_{s}+q_{0} I_{3}
\end{array}\right] * \omega_{s},
$$

where $\omega_{s}$ denotes the angular attitude velocity of the spacecraft relative to the inertial system and $\widetilde{\mathbf{q}}_{s}$ represents the skew symmetric matrix of $\mathbf{q}_{s}$ such that

$$
\tilde{q}_{s}=\left[\begin{array}{ccc}
0 & -q_{3} & q_{2} \\
q_{3} & 0 & -q_{1} \\
-q_{2} & q_{1} & 0
\end{array}\right] .
$$

The desired orbital coordinate system for the spacecraft tracking system is denoted as $R_{o}$, and $Q_{d}$ is the expected attitude quaternion. Thus, $\mathbf{Q}_{d}=\left[\begin{array}{ll}q_{d 0} & \mathbf{q}_{d}^{T}\end{array}\right]^{T}$, and the kinematic equation is defined as

$$
\dot{\mathbf{Q}_{d}}=\frac{1}{2} E\left(\mathbf{q}_{d}\right) * \omega_{d},
$$

where $\omega_{d}$ is the desired angular velocity from $R_{o}$ relative to $R$. In addition, the attitude tracking error quaternion is defined as $\mathbf{Q}_{e}=\left[\begin{array}{ll}q_{e 0} & \mathbf{q}_{e}^{T}\end{array}\right]^{T}$ such that

$$
\mathbf{Q}_{e}=\left[\begin{array}{c}
q_{e 0} \\
\mathbf{q}_{e}
\end{array}\right]=\left[\begin{array}{c}
q_{s 0} q_{d 0}+\mathbf{q}_{d}^{T} \mathbf{q}_{s} \\
q_{d 0} \mathbf{q}_{s}+\widetilde{\mathbf{q}}_{s} \mathbf{q}_{d}-q_{s 0} \mathbf{q}_{d}
\end{array}\right],
$$

which describes the error $R_{s}$ relative to $R_{o}$.

The principle of two vector attitude determinations was used to determine the desired quaternion of the spacecraft. The desired quaternion could then be used to obtain the attitude transformation matrix of the spacecraft orbit $R_{o}$ relative to the inertial orbit $R$. According to the known position vector $\mathbf{X}$ and velocity vector $\mathbf{V}$ of the spacecraft, matrix $B$ is the projection of $\mathbf{X}$ and $\mathbf{V}$ of the spacecraft in $R$, and matrix $A$ is the projection of $\mathbf{X}$ and $\mathbf{V}$ of the spacecraft in $R_{o}$. The unique transformation matrix is then defined as

$$
C_{B A}=B^{-1} A,
$$

where the matrixes $B$ and $A$ can be defined as

$$
\begin{aligned}
& B=\left[\frac{\mathbf{X}}{|\mathbf{X}|}, \frac{\mathbf{X} \times \mathbf{V}}{|\mathbf{X} \times \mathbf{V}|}, \frac{\mathbf{X} \times(\mathbf{X} \times \mathbf{V})}{|\mathbf{X} \times(\mathbf{X} \times \mathbf{V})|}\right], \\
& A=\left[\begin{array}{ccc}
0 & 0 & -1 \\
0 & -1 & 0 \\
-1 & 0 & 0
\end{array}\right] .
\end{aligned}
$$

Thus, the desired quaternion can be obtained as follows:

$$
\begin{aligned}
& q_{0}=\frac{1}{2} \sqrt{1+C_{B A}(1,1)+C_{B A}(2,2)+C_{B A}(3,3)}, \\
& q_{1}=\frac{1}{4 q_{0}}\left(C_{B A}(2,3)-C_{B A}(3,2)\right) \\
& q_{2}=\frac{1}{4 q_{0}}\left(C_{B A}(3,1)-C_{B A}(1,3)\right) \\
& q_{3}=\frac{1}{4 q_{0}}\left(C_{B A}(1,2)-C_{B A}(2,1)\right)
\end{aligned}
$$




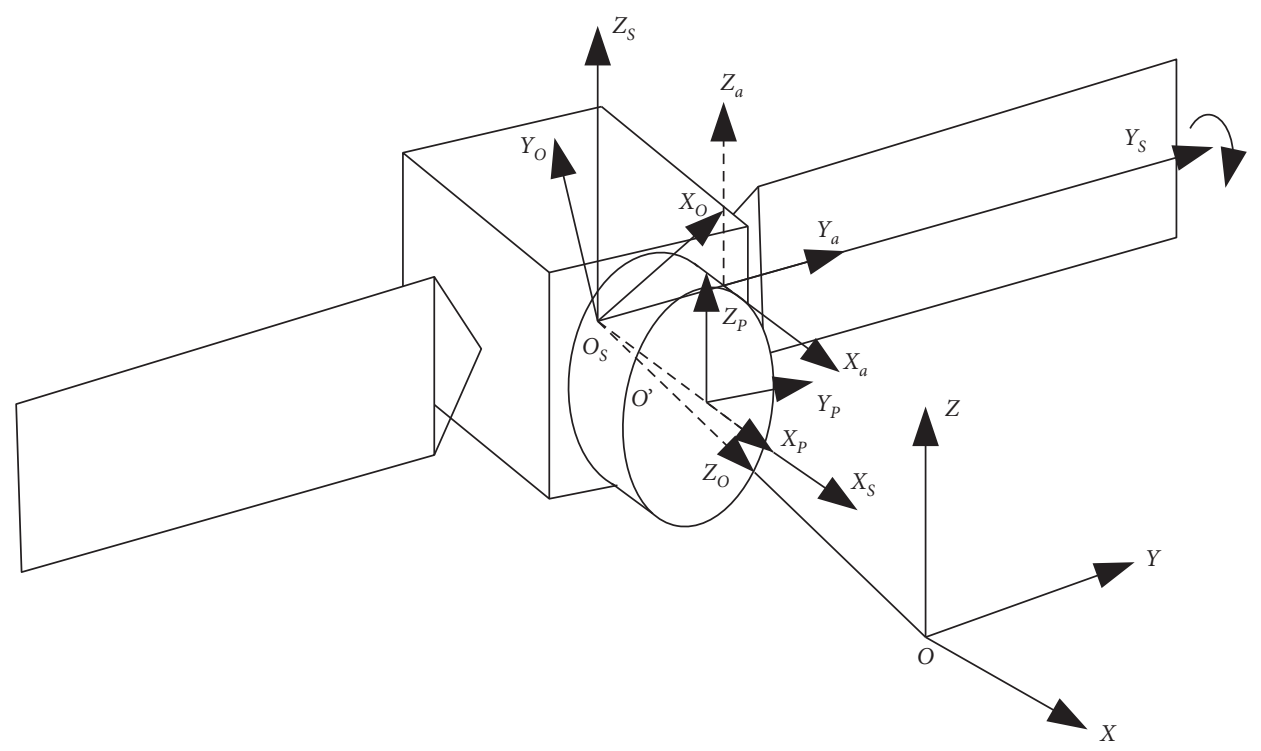

Figure 1: Coordinate system of spacecraft with appendages.

where $C_{s d}$ is the coordinate transformation matrix from $R_{o}$ to $R_{s}$, which can be calculated as

$$
C_{s d}=\left(q_{e 0}^{2}-\mathbf{q}_{e}^{T} \mathbf{q}_{e}\right) I_{3}+2 \mathbf{q}_{e} \mathbf{q}_{e}^{T}-2 q_{e 0} \widetilde{\mathbf{q}}_{e} .
$$

The angular attitude velocity tracking error of the spacecraft is defined as

$$
\omega_{e}=\omega_{s}-C_{s d} \omega_{d} .
$$

The high-inertia component is the rotation around the $\mathbf{x}_{s}$-axis of the spacecraft platform with the desired attitude angular velocity $\omega_{p d}=\left[\omega_{p d}, 0,0\right]^{T}$. Similarly, the flexible solar panels rotate around the $\mathbf{y}_{s}$-axis of the spacecraft platform with the desired attitude angular velocity $\omega_{a d}=\left[0, \omega_{a d}, 0\right]^{T}$. The kinematic equation for the desired attitude of the high-inertia components is $\dot{Q}_{p d}=(1 / 2) E\left(\mathbf{q}_{p d}\right) \omega_{p d}$ and that for the flexible solar panels is $\dot{Q}_{a d}=(1 / 2) E\left(\mathbf{q}_{a d}\right) \omega_{a d}$. Thus, the real kinematic equations are $\dot{Q}_{p}=(1 / 2) E\left(\mathbf{q}_{p}\right) \omega_{p}$ and $\dot{Q}_{a}=(1 / 2) E\left(\mathbf{q}_{a}\right) \omega_{a}$. The quaternion for the attitude tracking error can be obtained using (4), such that the attitude tracking error of the flexible solar panels is

$$
\mathbf{Q}_{a e}=\left[\begin{array}{c}
q_{a e 0} \\
\mathbf{q}_{a e}
\end{array}\right]=\left[\begin{array}{c}
q_{a 0} q_{a d 0}+\mathbf{q}_{a d}^{T} \mathbf{q}_{a} \\
q_{a d 0} \mathbf{q}_{a}+\widetilde{\mathbf{q}}_{a} \mathbf{q}_{a d}-q_{a 0} \mathbf{q}_{a d}
\end{array}\right] .
$$

The angular attitude velocity tracking error of the high-inertia components and flexible solar panels can be, respectively, expressed as

$$
\begin{aligned}
& \boldsymbol{\omega}_{p e}=\boldsymbol{\omega}_{p}-\boldsymbol{\omega}_{p d}, \\
& \boldsymbol{\omega}_{a e}=\boldsymbol{\omega}_{a}-\boldsymbol{\omega}_{a d} .
\end{aligned}
$$

When the high-inertia components and the flexible solar panels rotate only around the $\mathbf{x}_{s}$-axis and $\mathbf{y}_{s}$-axis, respectively, of the spacecraft platform, the rotational angle of the former is set as $\theta_{p} \in[0,2 \pi]$ and that of the latter is set as $\theta_{a} \in[0,2 \pi]$. The rotational angles can be calculated by

$$
\begin{aligned}
\boldsymbol{\theta}_{p} & =\int \boldsymbol{\omega}_{p} \mathrm{~d} t, \\
\boldsymbol{\theta}_{a i} & =\int \boldsymbol{\omega}_{a i} \mathrm{~d} t .
\end{aligned}
$$

The transformation matrixes from $R_{p}$ and $R_{a}$ to $R_{s}$ are then defined as

$$
\begin{aligned}
C_{p s} & =\left[\begin{array}{ccc}
1 & 0 & 0 \\
0 & \cos \left(\theta_{p x}\right) & \sin \left(\theta_{p x}\right) \\
0 & -\sin \left(\theta_{p x}\right) & \cos \left(\theta_{p x}\right)
\end{array}\right], \\
C_{a b i} & =\left[\begin{array}{ccc}
\cos \left(\theta_{a y i}\right) & 0 & -\sin \left(\theta_{a y i}\right) \\
0 & 1 & 0 \\
\sin \left(\theta_{a y i}\right) & 0 & \cos \left(\theta_{a y i}\right)
\end{array}\right] .
\end{aligned}
$$

2.2. Dynamics of the Spacecraft System. We considered the rotation of the rigid high-inertia components and flexible solar panels on the rigid-flexible coupling spacecraft, as well as the translation and rotation of the spacecraft platform and the modal vibration of the solar panels. The Lagrange method was used to deduce the complex equations and the dynamic model equations of the spacecraft system attitude, as follows:

$$
\left(M+m_{p}+2 m_{a}\right) \ddot{X}=\mathbf{P}_{s},
$$

$$
\begin{gathered}
\mathbf{J}_{s} \dot{\omega}_{s}+\widetilde{\boldsymbol{\omega}}_{s}\left(\mathbf{J}_{s} \boldsymbol{\omega}_{s}+\mathbf{R}_{s p} \boldsymbol{\omega}_{p}+\mathbf{R}_{s a} \boldsymbol{\omega}_{a}\right)+\mathbf{F}_{s a} \ddot{\eta}_{a i} \\
+\mathbf{R}_{s a} \dot{\omega}_{a i}+\mathbf{R}_{s p} \dot{\omega}_{p}=\mathbf{T}_{s}+\mathbf{T}_{s d p}+\mathbf{d},
\end{gathered}
$$

$$
\mathbf{J}_{a i} \dot{\omega}_{a i}+\mathbf{F}_{a i} \ddot{\eta}_{a i}+\mathbf{R}_{s a i}^{T} \dot{\omega}_{s}=\mathbf{T}_{a i}(i=-y, y),
$$

$\ddot{\eta}_{a i}+2 \zeta_{a i} \boldsymbol{\Omega}_{a i} \dot{\eta}_{a i}+\boldsymbol{\Lambda}_{a i} \boldsymbol{\eta}_{a i}+\mathbf{R}_{s a}^{T} \dot{\omega}_{s}+\mathbf{F}_{a i}^{T} \dot{\omega}_{a i}=0(i=-y,+y)$, 
where $M$ is the mass of the spacecraft platform; $m_{p}$ is the mass of the high-inertia component; $m_{a}$ is the mass of a single flexible solar panel; $P_{s}$ denotes the combined external forces on the spacecraft system; the inertia matrix with respect to $R_{s}$ of the spacecraft is defined as $J_{s}$ and satisfies the equation $J_{s}=J_{s 0}+C_{s a} J_{a 0} C_{s a}^{T}+C_{s p} J_{p} C_{s p}^{T} ; J_{a 0}$ is the inertia matrix of the flexible solar panel relative to the platform when it is not rotating; $J_{p}$ is the inertia matrix of the highinertia rotating component with respect to the platform; $T_{s}$ and $T_{a}$ denote the torque of the spacecraft platform and the solar panels, respectively; $T_{d}$ is the unknown external disturbance torque; $T_{s d p}$ is the sum of the dynamic and static unbalanced torques and the bearing torque of high-inertia components under the influence of coupling; $\eta_{a}$ denotes the modal coordinates of the solar panel; $\Omega_{a}$ is the diagonal matrix of the modal frequency of the solar panel; $\Lambda_{a}$ is the stiffness matrix of the solar panel such that $\Lambda_{a}=\Omega_{a}^{2}$; $\xi_{a}$ is the damping ratio of the solar panel. Notably, all these parameters are diagonal matrixes. From (14a)-(14b), $\mathbf{F}_{s a}=$ $\mathbf{F}_{s a_{-} \text {right }}+\mathbf{F}_{s a_{-} \text {left }}$ and $\mathbf{R}_{s a}=\mathbf{R}_{s a_{-} \text {right }}+\mathbf{R}_{s a_{-} \text {left }}$.

The connection point between the high-inertia rotation component and the platform was modeled as shown in Figure 1. In this paper, $\mathbf{r}_{s}$ denotes the vector from the connection point to the origin of the high-inertia rotation component's $R_{s}$ coordinate system; $\mathbf{r}_{p}$ denotes the vector from the origin of the $R_{p}$ coordinate system to the connection point; and $\mathbf{r}$ denotes the vector from the connection point to the mass element $d m$ on the high-inertia rotation component. When the high-inertia rotation component rotates around the $x$-axis at the angular velocity $\omega_{p}$, the unbalanced torque $\mathbf{T}_{s d}$ can be generated on the satellite platform. The dynamic equations for a high-inertia appendage are as follows:

$$
\begin{aligned}
\mathbf{T}_{s d}= & -\mathbf{r}_{p} \times \int_{p}\left[\boldsymbol{\omega}_{p} \times\left(\boldsymbol{\omega}_{p} \times \mathbf{r}\right)\right] \mathrm{d} m-\mathbf{r} \\
& \times \int_{p}\left[\boldsymbol{\omega}_{p} \times\left(\boldsymbol{\omega}_{p} \times \mathbf{r}\right)\right] \mathrm{d} m \\
= & -\boldsymbol{\omega}_{p} \times\left(\mathbf{J}_{p} \boldsymbol{\omega}_{p}\right)+\mathbf{m}_{p} \boldsymbol{\omega}_{p}^{T} \boldsymbol{\omega}_{p}\left(\mathbf{r}_{s} \times \mathbf{r}_{p}\right),
\end{aligned}
$$

$\mathbf{J}_{p} \dot{\omega}_{p}+\mathbf{R}_{s p}^{T} \dot{\omega}_{s}=\mathbf{T}_{p}$,

$$
\mathbf{T}_{s d p}=\mathbf{T}_{s d}-\mathbf{T}_{p},
$$

where $T_{p}$ is the control torque of the high-inertia component. Because the solar panels only rotate about the $y$-axis relative to the spacecraft platform, $\mathbf{F}_{s a}$ denotes the coupling coefficient matrix of the rotation of the spacecraft platform with the vibration of the solar panels such that

$$
F_{s a}=\left[f_{s a 1}\left[\begin{array}{c}
\sin \left(\theta_{a y}\right) \\
1 \\
\cos \left(\theta_{a y}\right)
\end{array}\right], f_{s a 2}\left[\begin{array}{c}
\sin \left(\theta_{a y}\right) \\
1 \\
\cos \left(\theta_{a y}\right)
\end{array}\right], \ldots, f_{s a 6}\left[\begin{array}{c}
\sin \left(\theta_{a y}\right) \\
1 \\
\cos \left(\theta_{a y}\right)
\end{array}\right]\right. \text {, }
$$

where $f_{s a}$ is the coefficient matrix of the platform rotation to the solar panel vibration, which is related to the rotating angle $\theta_{\text {ay }}, \mathbf{F}_{a}$ is the coupling coefficient matrix between the vibration and rotation of solar panels, $\mathbf{R}_{s a}$ is the coupling coefficient matrix of rotation between the solar panels and the platform and is calculated in a similar manner as equation (18), with $r_{s a}$ as the angle-independent coefficient, and $\mathbf{R}_{s p}$ is the matrix of vibration of the high-inertia component with the rotation of the spacecraft platform. The high-inertia component rotates only around the $x$-axis relative to the spacecraft platform; thus, $\mathbf{R}_{s p}$ can be described by a variation of (16).

Equation (13) shows the dynamic equations of the spacecraft system, including the translation of the platform, as well as the rotation of the high-inertia components and solar panels. Upon substituting (5) and (11) into (14a)-(14d), we obtain

$$
\Theta \dot{\omega}+\Gamma+\Delta=\mathbf{T},
$$

where

$$
\begin{aligned}
& \boldsymbol{\omega}=\left[\begin{array}{ll}
\boldsymbol{\omega}_{e} & \boldsymbol{\omega}_{a e}
\end{array}\right]^{T}, \\
& \boldsymbol{\Theta}=\left[\begin{array}{cc}
\mathbf{J}_{s 0}-\mathbf{F}_{s a} \mathbf{F}_{s a}^{T} & \mathbf{R}_{s a}-\mathbf{F}_{s a} \mathbf{F}_{a}^{T} \\
\mathbf{R}_{s a}^{T}-\mathbf{F}_{a} \mathbf{F}_{s a}^{T} & \mathbf{J}_{a}-\mathbf{F}_{a} \mathbf{F}_{a}^{T}
\end{array}\right] \\
& \boldsymbol{\Gamma}=\left[\begin{array}{c}
\mathbf{R}_{s p} \dot{\omega}_{P}+\boldsymbol{\omega}_{s} \times\left(\mathbf{J}_{s 0} \boldsymbol{\omega}_{s}+\mathbf{R}_{s p} \boldsymbol{\omega}_{p}+\mathbf{R}_{s a} \boldsymbol{\omega}_{a}\right)+\left(\mathbf{J}_{s 0}-\mathbf{F}_{s a} \mathbf{F}_{s a}^{T}\right)\left(-\widetilde{\boldsymbol{\omega}}_{e} \mathbf{C}_{s d} \boldsymbol{\omega}_{d}+\mathbf{C}_{s d} \dot{\omega}_{d}\right)-\mathbf{T}_{s d p} \\
\mathbf{R}_{s a}^{T}-\mathbf{F}_{a} \mathbf{F}_{s a}^{T}\left(-\widetilde{\boldsymbol{\omega}}_{e} \mathbf{C}_{s d} \boldsymbol{\omega}_{d}+\mathbf{C}_{s d} \dot{\omega}_{d}\right)
\end{array}\right], \\
& \boldsymbol{\Delta}=\left[\begin{array}{c}
\Delta_{1} \\
\Delta_{2}
\end{array}\right]=\left[\begin{array}{c}
\mathbf{F}_{s a}\left(2 \boldsymbol{\zeta}_{a i} \boldsymbol{\Omega}_{a i} \dot{\eta}_{a i}+\boldsymbol{\Lambda}_{a i} \boldsymbol{\eta}_{a i}\right)+\left(C_{s a} \mathbf{J}_{a 0} C_{s a}^{T}+C_{s p} \mathbf{J}_{p} C_{s p}^{T}\right)\left(\dot{\omega}_{e}-\widetilde{\boldsymbol{\omega}}_{e} C_{s d} \boldsymbol{\omega}_{d}+C_{s d} \dot{\omega}_{d}\right)-\mathbf{d}+\Delta\left(\mathbf{F}_{s a}, \mathbf{R}_{s a}\right) \\
\Delta\left(\mathbf{F}_{s a}, \mathbf{R}_{s a}\right)
\end{array}\right], \\
& \mathbf{T}=\left[\begin{array}{c}
\mathbf{T}_{s} \\
\mathbf{T}_{a}
\end{array}\right] .
\end{aligned}
$$


Remark 1. The moment of inertia of the spacecraft system has the specific form $J=J_{0}+\Delta J$, where $J_{0}$ is the nonsingular nominal constant matrix, and $\Delta J$ is the uncertainty of moment of inertia and is a bounded constant.

Remark 2. $\left(\Delta F_{s a}, \Delta R_{s a}, \Delta R_{s p}\right)$ represent the combined uncertainties on parameters $F_{s a}, R_{s a}$, and $R_{s p}$; these uncertainties exist because, although the high-inertia components and solar panels rotate predominantly around one axis of the spacecraft, they exhibit small rotations around the other axes. Thus, the combined uncertainties are bounded.

Remark 3. The disturbance $\Delta$ is bounded, and satisfies $\Delta \leq \Delta_{d}$, where $d$ is a constant.

\section{Sliding Mode Control Law Design}

Lemma 1. The extended Lyapunov of finite-time stability can be expressed in the form of a fast TSM as follows [34, 35]:

$$
\dot{V}(x)+\lambda_{1} V(x)+\lambda_{2} V^{\gamma}(x) \geq 0 .
$$

If (18) satisfies the conditions $\lambda_{1}>0, \lambda_{2}>0$ and $0<\gamma<1$, the convergence time can be given by

$$
T \leq \frac{1}{\lambda_{1}(1-\gamma)} \ln \frac{\lambda_{1} V^{1-\gamma}\left(x_{0}\right)+\lambda_{2}}{\lambda_{2}} .
$$

Lemma 2. For any $x_{i} \in R(i=1,2, \ldots, n)$ and a positive real number $\gamma \in(0,1)$, the following inequality holds [36]:

$$
\left(\sum_{i=1}^{n}\left|x_{i}\right|^{2}\right)^{(1+\gamma / 2)} \leq \sum_{i=1}^{n}\left|x_{i}\right|^{1+\gamma} \text {. }
$$

Lemma 3. We consider the system $\dot{x}=f(x, u)$, where $f$ is a continuous function that satisfies $f(0)=0$, and a Lyapunov function $V(x)$ that satisfies [37]

$$
\dot{V}(x)+\lambda V^{\alpha}(x) \leq 0,
$$

where $\lambda>0,0<\alpha<1$. Then, the origin is a finite-time stable equilibrium of the system $\dot{x}=f(x, u)$. Let T represent the time required for the system to reach $V(x) \equiv 0$. If $\mathbf{x}_{0}$ is the initial system state, the convergence time satisfies

$$
T \leq \frac{V^{1-\alpha}\left(\mathbf{x}_{0}\right)}{\lambda(1-\alpha)} .
$$

Using the quaternion for the attitude tracking error of the spacecraft platform and solar panel to describe the attitude system state, we can write $\mathbf{x}=\left[\begin{array}{ll}\mathbf{q}_{e} & \mathbf{q}_{a e}\end{array}\right]^{T}$ and define

$$
\dot{x}=\left[\dot{q}_{e} \dot{q}_{a e}\right]^{T}=\frac{1}{2} \mathbf{E} \boldsymbol{\omega} .
$$

Thus, $\ddot{x}$ can be given as

$$
\ddot{x}=\frac{1}{4} \mathbf{L}+\frac{1}{2}(\mathbf{E} \Theta)(\mathbf{T}-\boldsymbol{\Gamma}-\mathbf{\Delta}) .
$$

Finally, the augmented matrix $\mathbf{E}$ can be written as $\mathbf{E}=$ $\left[\begin{array}{cc}\mathbf{E}\left(\mathbf{q}_{e}\right) & 0 \\ 0 & \mathbf{E}\left(\mathbf{q}_{a e}\right)\end{array}\right]$ and $\mathbf{L}=\left[\begin{array}{c}\mathbf{q}_{e} \omega_{e}^{T} \omega_{e} \\ \mathbf{q}_{a e} \omega_{a e}^{T} \omega_{a e}\end{array}\right]$

\subsection{Controller Design of the Spacecraft Platform and Solar Panel}

3.1.1. Integral Sliding Mode Surface Design. The integral sliding mode surface can be defined by

$$
\mathbf{S}=\mathbf{k}_{x} \dot{\mathbf{x}}+\int_{0}^{t} \mathbf{C}_{1} \mathbf{S}_{1}\left(\boldsymbol{\lambda}_{1}, \boldsymbol{\rho}_{1}, \mathbf{x}, \boldsymbol{\varepsilon}_{1}\right)+\mathbf{C}_{2} \mathbf{S}_{2}\left(\boldsymbol{\lambda}_{2}, \boldsymbol{\rho}_{2}, \dot{\mathbf{x}}, \boldsymbol{\varepsilon}_{2}\right) \mathrm{d} t,
$$

where $\mathbf{C}_{1}=\operatorname{diag}\left(c_{11}, c_{12}, c_{13}, c_{14}, c_{15}, c_{16}\right)$ and $\mathbf{C}_{2}=\operatorname{diag}$ $\left(c_{21}, c_{22}, c_{23}, c_{24}, c_{25}, c_{26}\right)$, and

$$
\begin{aligned}
& \gamma_{1}=\left[\gamma_{11}, \gamma_{12}, \gamma_{13}, \gamma_{14}, \gamma_{15}, \gamma_{16}\right]^{T}, \\
& \gamma_{2}=\left[\gamma_{21}, \gamma_{22}, \gamma_{23}, \gamma_{24}, \gamma_{25}, \gamma_{26}\right]^{T}, \\
& \varepsilon_{1}=\left[\varepsilon_{11}, \varepsilon_{12}, \varepsilon_{13}, \varepsilon_{14}, \varepsilon_{15}, \varepsilon_{16}\right]^{T}, \\
& \varepsilon_{2}=\left[\varepsilon_{21}, \varepsilon_{22}, \varepsilon_{23}, \varepsilon_{24}, \varepsilon_{25}, \varepsilon_{26}\right]^{T},
\end{aligned}
$$

where $c_{1 i}>0$ and $c_{2 i}>0$ are the sliding surface design matrixes for $i=1,2, \ldots, 6$. Furthermore, the vectors $\mathbf{S}_{1}$ and $\mathbf{S}_{2}$ can be defined as

$$
\begin{aligned}
& \mathbf{S}_{1}=\left[S_{11}, S_{12}, S_{13}, S_{14}, S_{15}, S_{16}\right]^{T}, \\
& \mathbf{S}_{2}=\left[S_{21}, S_{22}, S_{23}, S_{24}, S_{25}, S_{26}\right]^{T} .
\end{aligned}
$$

In (30), $S_{1 i}$ and $S_{2 i}$ are defined as

$$
\begin{aligned}
& S_{1 i}\left(\gamma_{1 i}, \rho_{1 i}, x_{1 i}, \varepsilon_{1 i}\right)= \begin{cases}\left|x_{1 i}\right|^{\gamma_{1 i}}, & \text { if }\left|x_{1 i}\right| \leq \varepsilon_{1 i}, \\
\varepsilon_{1 i}^{\gamma_{1 i}}-\rho_{1 i}\left|x_{1 i}\right|^{\rho_{1 i}} \operatorname{sign}\left(x_{1 i}\right), & \text { if }\left|x_{1 i}\right|>\varepsilon_{1 i},\end{cases} \\
& S_{2 i}\left(\gamma_{2 i}, \rho_{2 i}, \dot{x}_{2 i}, \varepsilon_{2 i}\right)= \begin{cases}\left|\dot{x}_{2 i}\right|^{\gamma_{2 i}}, & \text { if }\left|\dot{x}_{2 i}\right| \leq \varepsilon_{2 i}, \\
\varepsilon_{2 i}{ }^{2 i} \rho_{2 i}\left|\dot{x}_{2 i}\right|^{\rho_{2 i}} \operatorname{sign}\left(\dot{x}_{2 i}\right), & \text { if }\left|\dot{x}_{2 i}\right|>\varepsilon_{2 i},\end{cases}
\end{aligned}
$$

where $\gamma_{1 i}$ and $\gamma_{2 i}$ are the positive constant gains, which satisfy $0<\gamma_{2 i}<1$ and $\gamma_{1 i}=\left(\gamma_{2 i} / 2-\gamma_{2 i}\right)$. In the same way, $\rho_{1 i}$, $\rho_{2 i}, \varepsilon_{1 i}$, and $\varepsilon_{2 i}$ are the positive constant gains, which satisfy $\rho_{1 i} \geq 1, \rho_{2 i} \geq 1, \varepsilon_{1 i}>0$, and $\varepsilon_{2 i}>0$, respectively.

The control torque $\mathbf{T}$ can be expressed as follows:

$$
\begin{aligned}
\mathbf{T}= & 2\left(\mathbf{K}_{x} \mathbf{E} \Theta\right)^{-1}\left(-\boldsymbol{\tau} \mathbf{S}-\mathbf{K}_{s} \operatorname{sgn}(\mathbf{S})-\mathbf{C}_{1} \mathbf{S}_{1}\left(\boldsymbol{\gamma}_{1}, \boldsymbol{\rho}_{1}, \mathbf{x}, \boldsymbol{\varepsilon}_{1}\right)\right. \\
& \left.-\mathbf{C}_{2} \mathbf{S}_{2}\left(\boldsymbol{\gamma}_{2}, \boldsymbol{\rho}_{2}, \dot{x}, \boldsymbol{\varepsilon}_{2}\right)\right)+\frac{1}{2}(\mathbf{E} \Theta)^{-1} \mathbf{L}+\boldsymbol{\Gamma}+\boldsymbol{\Delta} .
\end{aligned}
$$

Remark 4. From (28), it is observed that the controller parameters $\gamma_{1 i}, \gamma_{2 i}, k_{x i}, C_{1 i}$, and $C_{2 i}$ affect the convergences performances of the closed-loop systems. Smaller $\gamma_{1 i}$ and $\gamma_{2 i}$ and larger $k_{x i}, C_{1 i}$, and $C_{2 i}$ will yield a more quick convergence of the attitude tracking errorx $(t)$; xcan be further reduced by increasing the control parameters $\tau$ and $k_{S}$. 
3.1.2. Proof. With the spacecraft attitude dynamics model described in (14a)-(14d), the integral sliding mode (see (28)) and the control law (see (32)) were designed. The system state will slide to the equilibrium point in a finite time after reaching the sliding mode surface. Based on Lyapunov theory, it can be proved that

$$
V=\frac{1}{2} \mathbf{S}^{T} \mathbf{S}
$$

because

$$
\begin{aligned}
\dot{\mathbf{S}}= & \mathbf{k}_{x} \ddot{x}+\mathbf{C}_{1} \mathbf{S}_{1}\left(\gamma_{1}, \boldsymbol{\rho}_{1}, \mathbf{x}, \boldsymbol{\varepsilon}_{1}\right)+\mathbf{C}_{2} \mathbf{S}_{2}\left(\gamma_{2}, \boldsymbol{\rho}_{2}, \dot{x}, \boldsymbol{\varepsilon}_{2}\right) \\
= & \mathbf{k}_{x}\left[\frac{1}{4} \mathbf{L}+\frac{1}{2}(\mathbf{E} \Theta)(\mathbf{T}-\boldsymbol{\Gamma}-\Delta)\right]+\mathbf{C}_{1} \mathbf{S}_{1}\left(\gamma_{1}, \boldsymbol{\rho}_{1}, \mathbf{x}, \boldsymbol{\varepsilon}_{1}\right) \\
& +\mathbf{C}_{2} \mathbf{S}_{2}\left(\gamma_{2}, \boldsymbol{\rho}_{2}, \dot{x}, \boldsymbol{\varepsilon}_{2}\right) .
\end{aligned}
$$

Differentiating $V$ with respect to time yields (30); from (32), it can be determined that

$$
\begin{aligned}
\dot{V}= & \mathbf{S}^{T} \dot{\mathbf{S}}=\mathbf{S}^{T}\left\{\mathbf{k}_{x}\left[\frac{1}{4} \mathbf{L}+\frac{1}{2}(\mathbf{E \Theta})(\mathbf{T}-\boldsymbol{\Gamma}-\boldsymbol{\Delta})\right]\right. \\
& \left.+\mathbf{C}_{1} \mathbf{S}_{1}\left(\gamma_{1}, \boldsymbol{\rho}_{1}, \mathbf{x}, \boldsymbol{\varepsilon}_{1}\right)+\mathbf{C}_{2} \mathbf{S}_{2}\left(\gamma_{2}, \boldsymbol{\rho}_{2}, \dot{x}, \boldsymbol{\varepsilon}_{2}\right)\right\} \\
= & \mathbf{S}^{T}\left[-\tau \mathbf{S}-\mathbf{K}_{s} \operatorname{sgn}(\mathbf{S})\right]=-\tau V-k_{s} V^{(1 / 2)} .
\end{aligned}
$$

According to Lemma 1, the convergence time can be determined as

$$
T \leq \frac{2}{\tau} \ln \frac{\tau V^{(1 / 2)}\left(x_{0}\right)+k_{s}}{k_{s}} .
$$

3.2. Controller Design for High-Inertia Components. The high-inertia components rotate around the $x$-axis at a certain attitude angular velocity relative to the spacecraft platform. Thus, a control law can be designed for these components.

3.2.1. Sliding Mode Surface Design. The sliding mode function is defined as

$$
S_{p}=K_{P} \omega_{P e}+K_{d} \operatorname{sig}^{\gamma}\left(\dot{\omega}_{P e}\right),
$$

where $K_{P}=\operatorname{diag}\left(k_{p 1}, k_{p 2}, k_{p 3}\right), K_{d}=\operatorname{diag}\left(k_{d 1}, k_{d 2}, k_{d 3}\right)$, and $\gamma>1$ is a positive odd number. When the sliding mode function $S_{p}=0$, the attitude angular velocity tracking error of the high-inertia component is equal to $0\left(\omega_{P e}=0\right)$ because

$$
\dot{\omega}_{P e}=\left|\frac{k_{p i}}{k_{d i}} \omega_{P e}\right|^{(1 / \gamma)} \operatorname{sign}\left(\frac{k_{p i}}{k_{d i}} \omega_{P e}\right)=\frac{k_{p i}}{k_{d i}}\left|\omega_{P e}\right|^{(1 / \gamma)} \operatorname{sign}\left(\omega_{P e}\right) .
$$

The control torque $\mathbf{T}_{p}$ can be expressed as

$$
\mathbf{T}_{p}=\mathbf{J}_{p}\left(\mathbf{k}_{p}\right)^{-1}\left(-\tau \mathbf{S}_{p}-k_{d} \operatorname{sig}^{\gamma}\left(\ddot{\omega}_{p e}\right)\right)+R_{s p}^{T} \dot{\omega}_{s} .
$$

3.2.2. Proof. As the high-inertia component dynamics model described in (16), the sliding mode (see (37)) was designed. The system state slides to the equilibrium point in a finite time after reaching the sliding mode surface. Based on Lyapunov theory, it can be proved that

$$
V_{2}=\frac{1}{2} \omega_{P e}^{T} J \omega_{P e}
$$

Differentiation of $V_{2}$ with respect to time yields

$$
\begin{aligned}
V_{2} & =\omega_{P e}^{T} J \omega_{P e} \sum_{1}^{3} \frac{k_{p i}}{k_{d i}} \omega_{P e}^{T} J \mid \omega_{P e i}{ }^{(1 / \gamma)} \operatorname{sign}\left(\omega_{P e i}\right) \\
& =-\sum_{1}^{3} \frac{k_{p i}}{k_{d i}} J\left|\omega_{P e i}^{2}\right|^{(\gamma+1 / 2 \gamma)} \\
& \leq-\min \left\{\frac{k_{p i}}{k_{d i}}\right\}\left(2 \cdot \frac{1}{2} J \sum_{1}^{3} \omega_{P e i}\right)^{(\gamma+1 / 2 \gamma)} \\
& =-\min \left\{\frac{k_{p i}}{k_{d i}}\right\} \cdot 2^{(\gamma+1 / 2 \gamma)} V^{(\gamma+1 / 2 \gamma)} .
\end{aligned}
$$
satisfies

Furthermore, letting $\xi=-\min \left\{k_{p i} / k_{d i}\right\} \cdot 2^{(\gamma+1 / 2 \gamma)}, \quad \dot{V}_{2}$

$$
\dot{V}_{2}+\xi V_{2}^{(\gamma+1 / 2 \gamma)} \leq 0
$$

Finally, the convergence time can be determined according to Lemma 3, as follows:

$$
T_{p} \leq \frac{2 \gamma V_{2}^{(\gamma+1 / 2 \gamma)}(0)}{\xi(\gamma-1)} .
$$

\section{Numerical Simulation Results}

This section presents numerical simulations to demonstrate the performance of the proposed nonsingular integral sliding mode controller (NISMC). The results of the proposed method are compared with those of the fast TSM finite-time controller (FSMC) designed by Yang and Yang in [38] and the PD controller. The simulation results of the spacecraft with high-inertia components and flexible solar panels are presented, and the proposed control laws are demonstrated to have superior performance. The fast-sliding mode finite-time controller exhibits higher convergence speed and an accurate attitude tracking and control of the component angle. In this analysis, the desired angular velocity of the flexible solar panels about the $y$-axis of the spacecraft was set as $\omega_{a d}=0.1 \mathrm{rad} / \mathrm{s}$ and that of the large rigid inertial component about the $x$-axis of the spacecraft as $\omega_{p d}=0.2 \mathrm{rad} / \mathrm{s}$. The expected attitude of the spacecraft platform is the coordinate system $R_{s}$ that coincides with $R_{o}$.

Figures 2 and 3 present the results of the vector component of the error quaternion and the error of the attitude angular velocity of the platform, respectively. Figure 4 presents the results of the vector component of the error quaternion of the flexible solar panels. Figures 5 and 6 present the results of the error of the attitude angular 


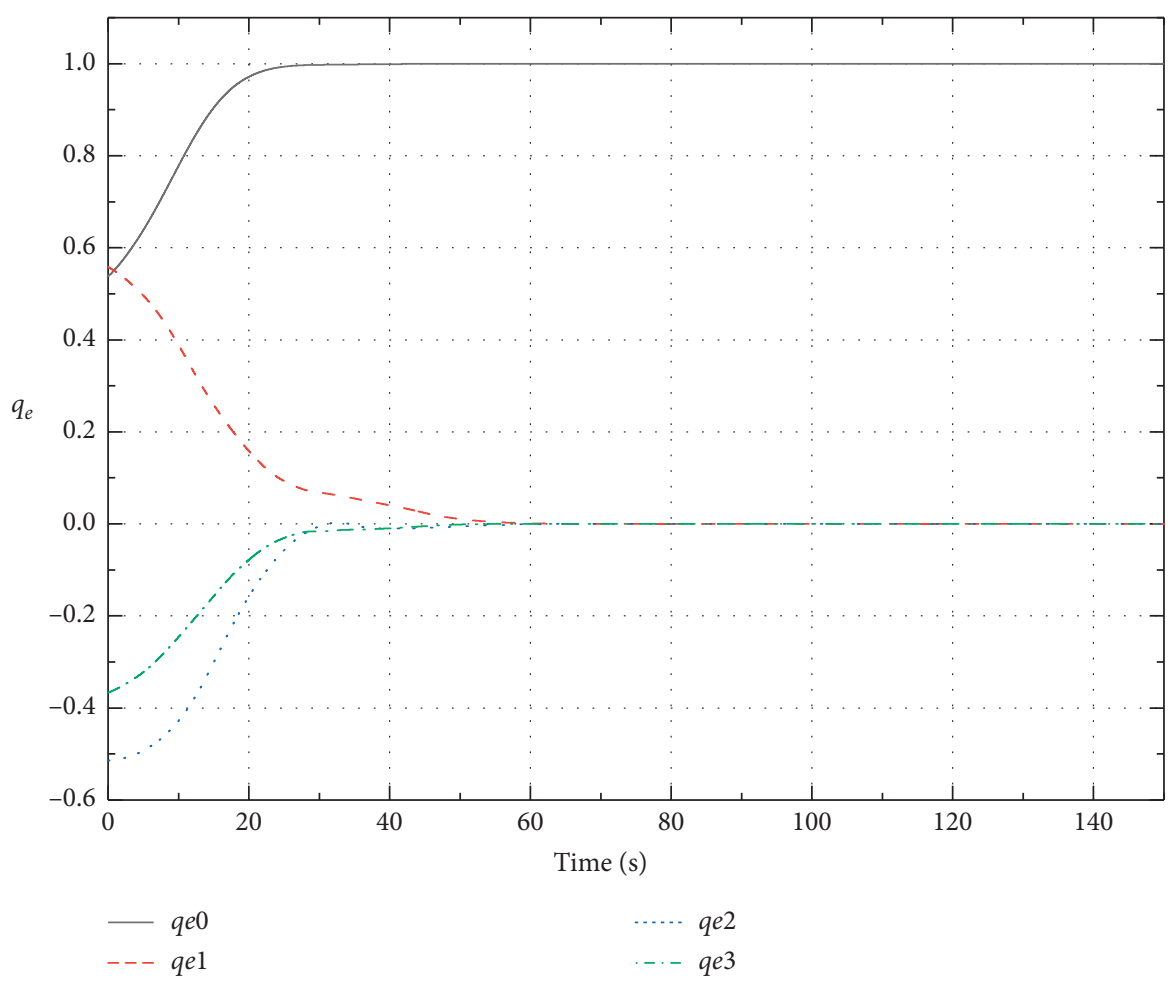

Figure 2: Trajectories of the vector component of the platform error quaternion.

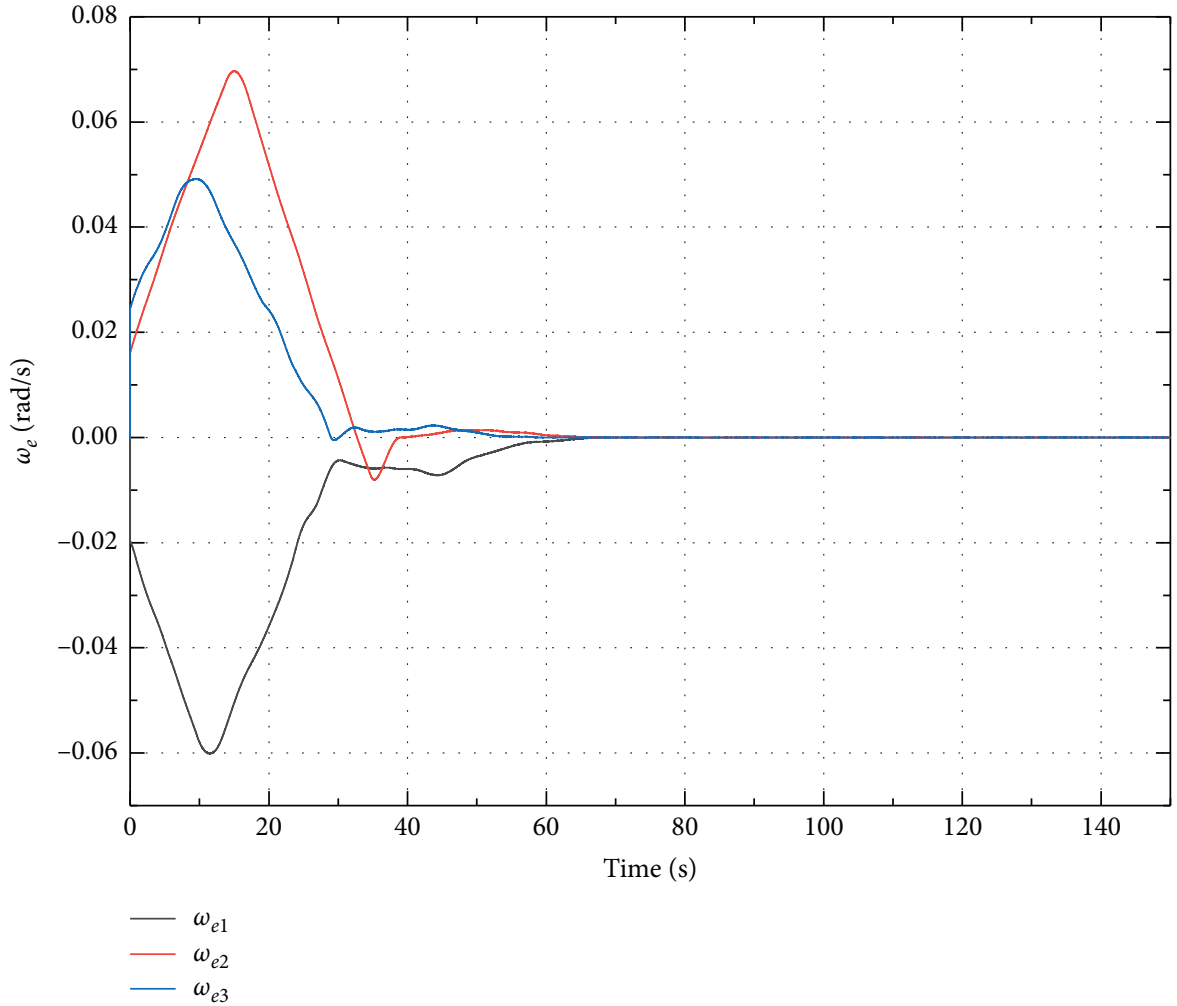

Figure 3: Trajectories of the error of attitude angular velocity of platform.

velocity and the attitude angular velocity of the flexible solar panels relative to the spacecraft platform. Figure 7 shows the attitude angular velocity and error of the angular velocity of the large rigid inertial component that rotates around the $x$-axis of the spacecraft platform. Figures 3, 5, and 7 indicate that the error of the attitude angular velocity 


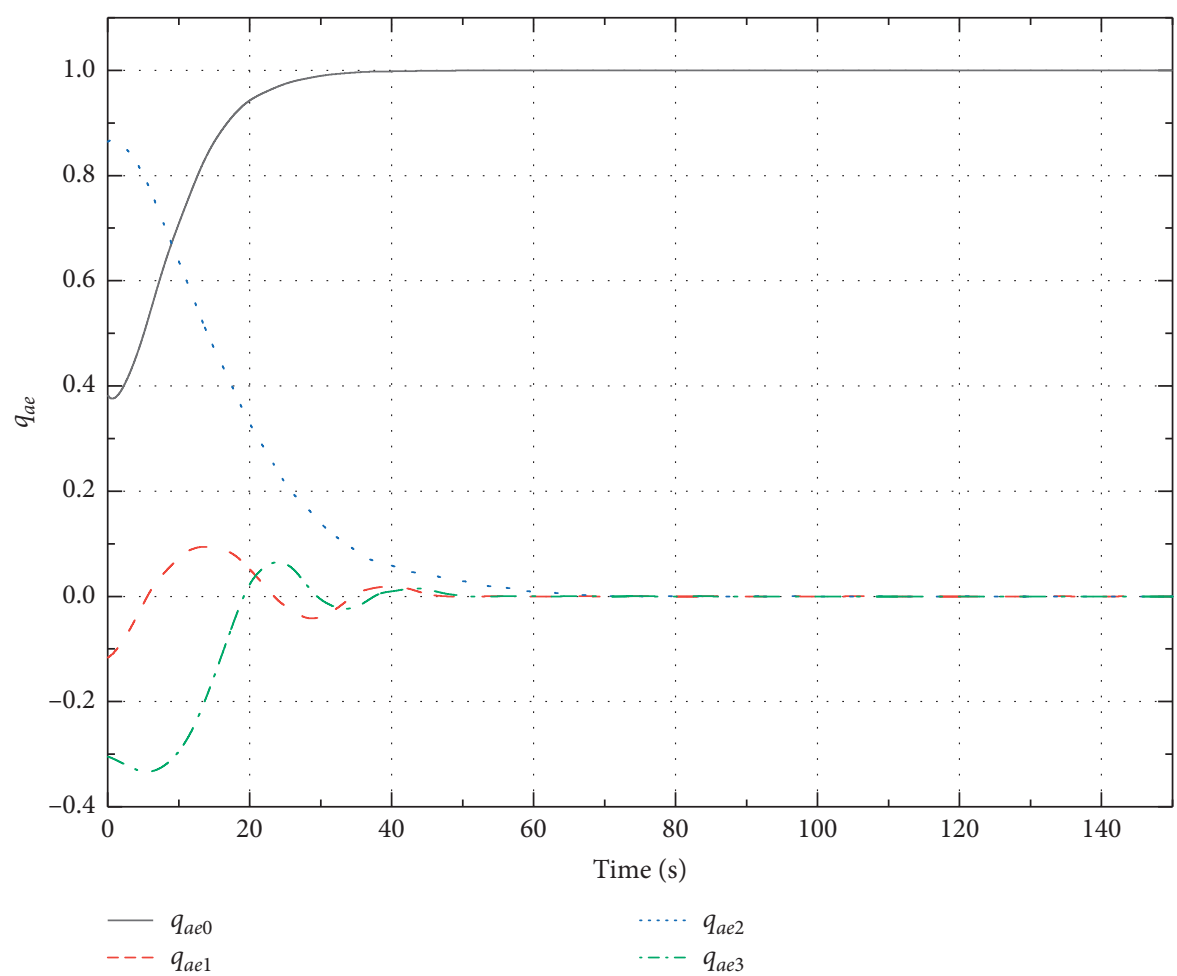

Figure 4: Trajectories of vector component of the error quaternion of flexible solar panel.

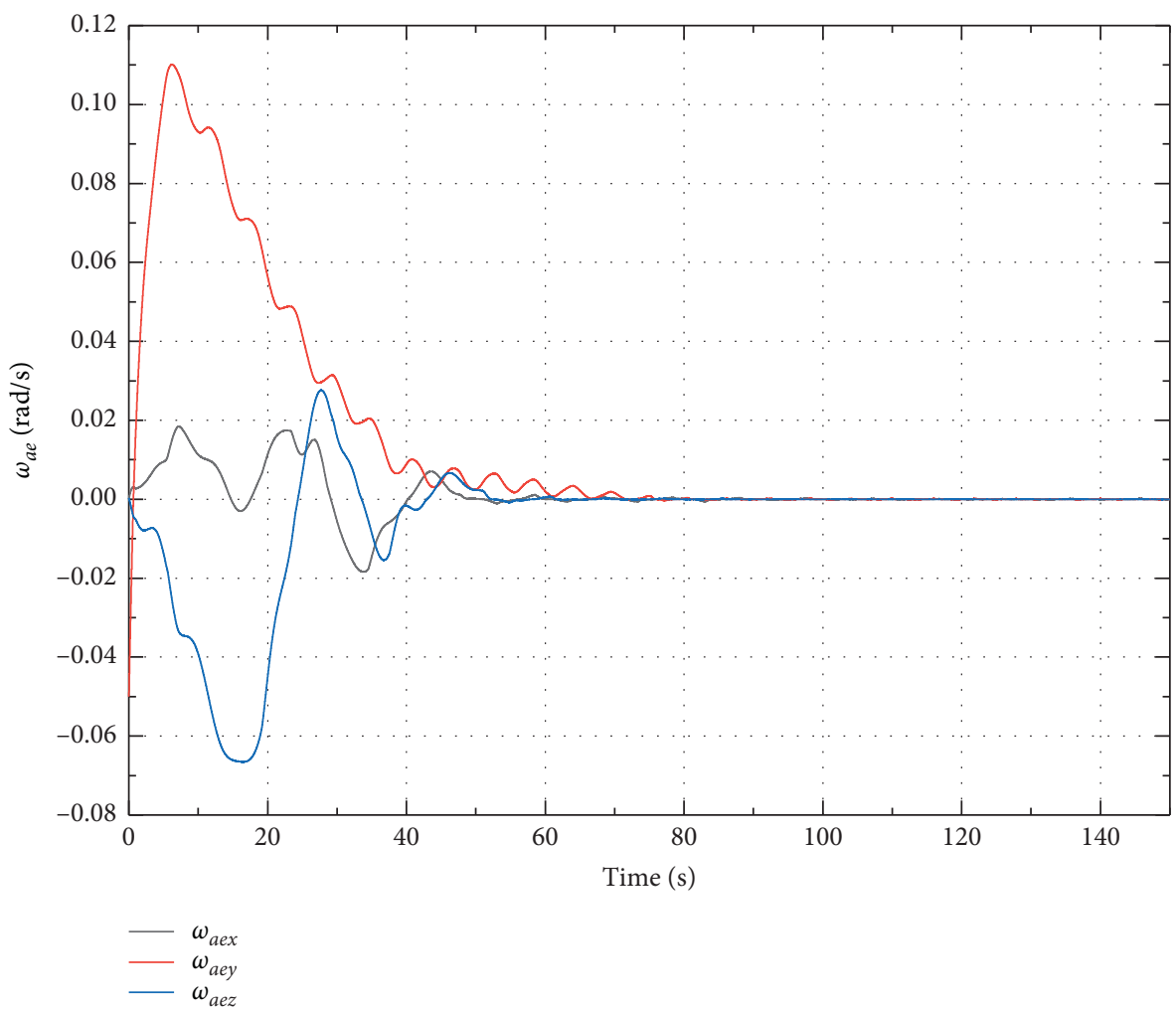

Figure 5: Trajectories of the error of attitude angular velocity of flexible solar panel.

of the spacecraft satisfies the constraint. Therefore, the system can reach the sliding surface in a finite time, and when the system enters the sliding surface, it can maintain sliding mode motion. Therefore, the designed controller can ensure that the system tracks the desired attitude angle and angular velocity. 


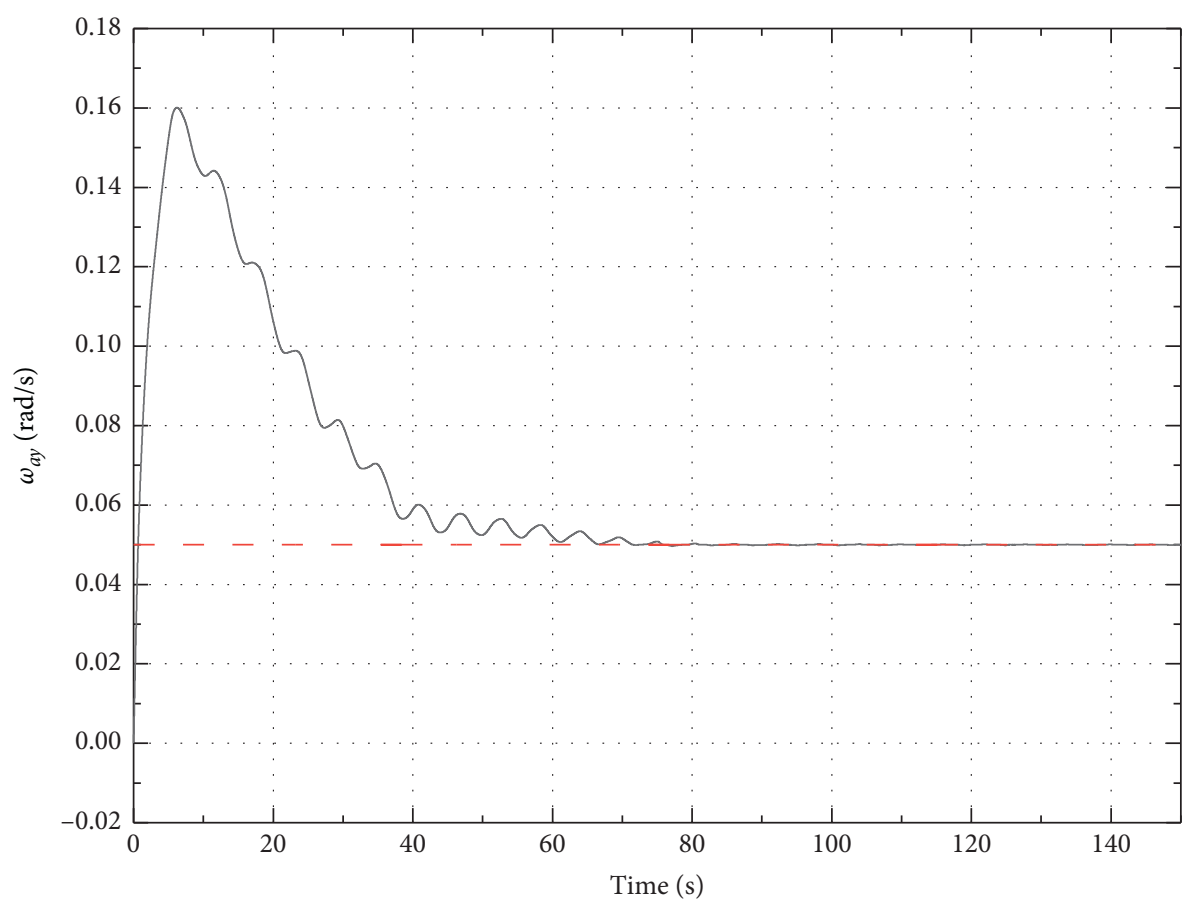

- Actual angular velocity

- - - Desired angular velocity

Figure 6: Trajectories of the $y$-axis flexible solar panel's attitude angular velocity.

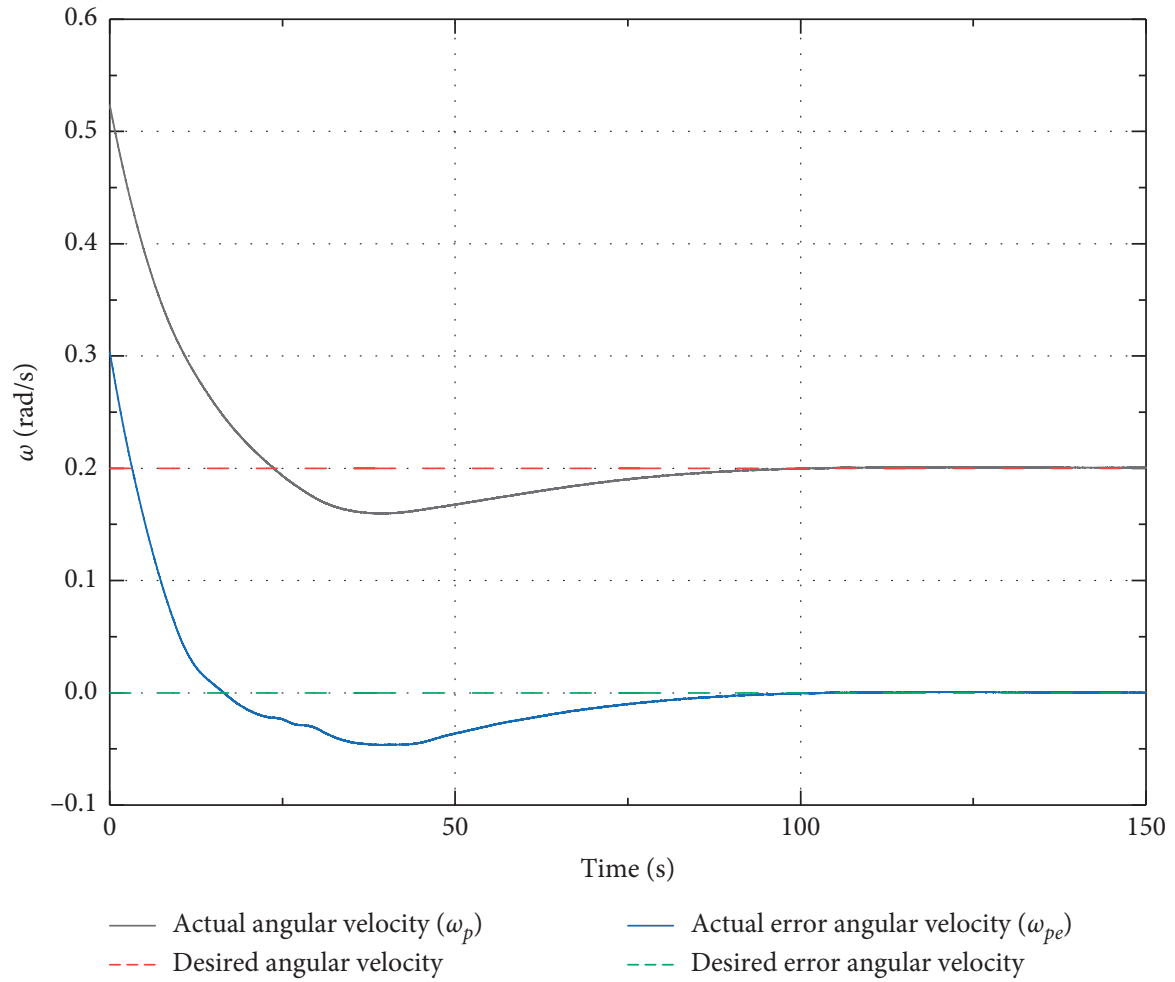

Figure 7: Trajectories of angular velocity and error of angular velocity of the large rigid inertial component.

Figures 8-11 present the results of the error quaternion and the error of angular velocity of the flexible solar panels and platform. These figures indicate that the proposed method converges faster than the existing methods (FSMC and PD). Table 1 presents the results of the stable error of attitude angular velocity and the stable error quaternion of 


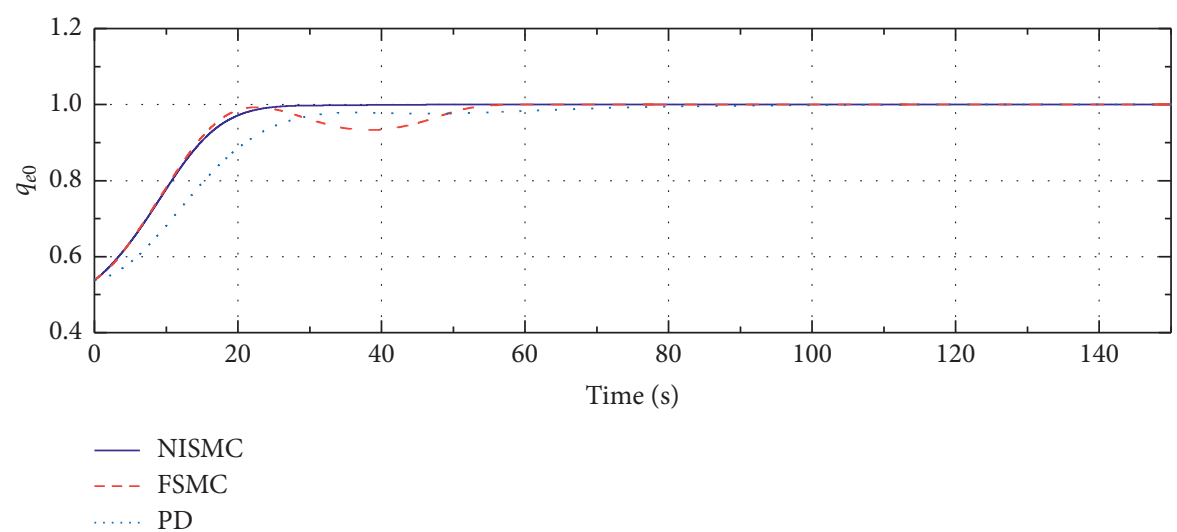

(a)

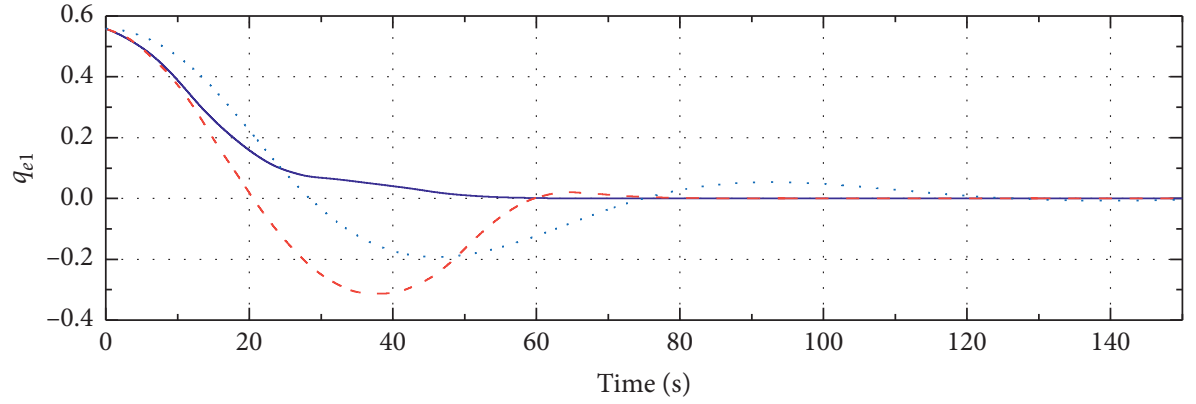

- NISMC

-. - FSMC

PD

(b)

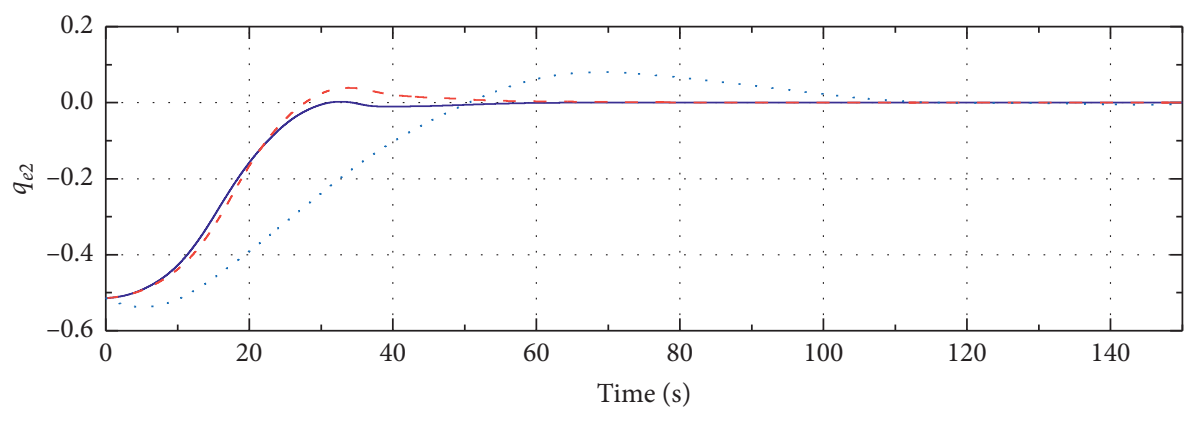

NISMC

FSMC

PD

(c)

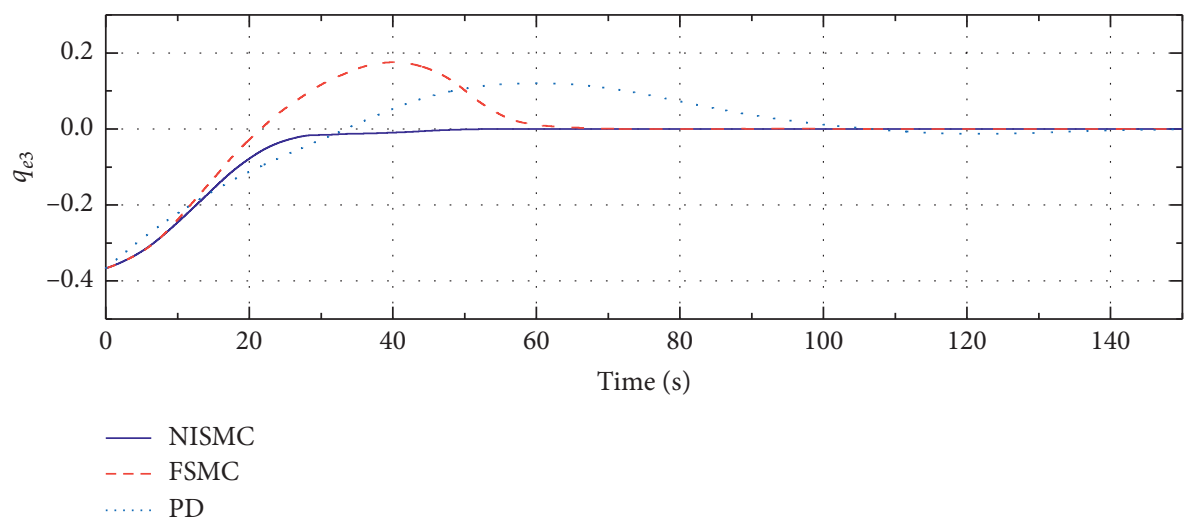

(d)

Figure 8: Comparison of platform error quaternions. 


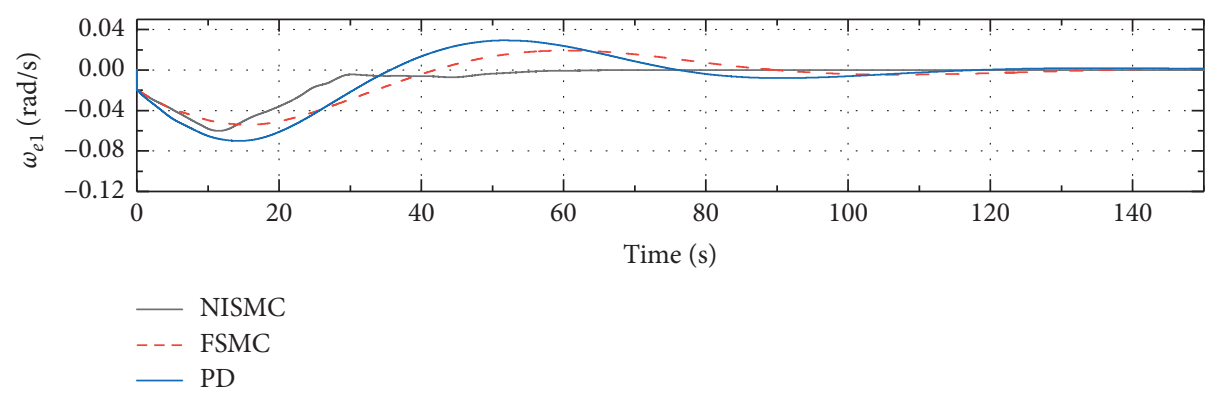

(a)

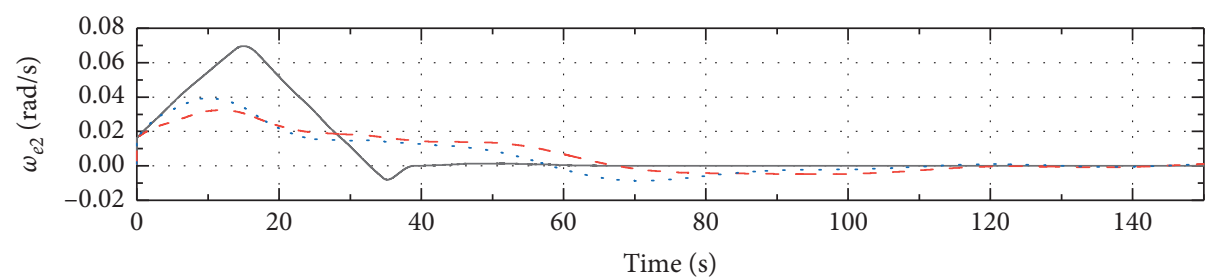

- NISMC

- - FSMC

$\mathrm{PD}$

(b)

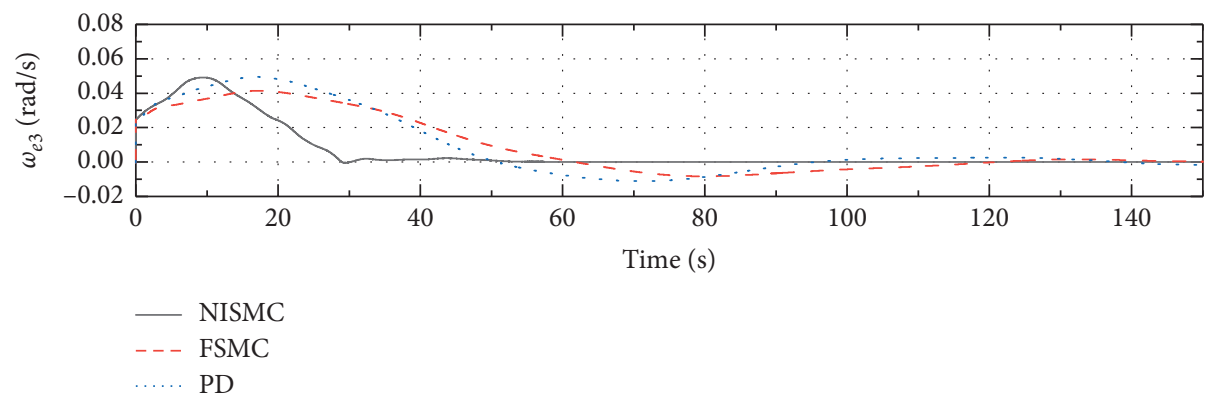

(c)

FIGURE 9: Comparison of errors of attitude angular velocity of platform.

the flexible solar panels and platform. The table indicates that the stable error of attitude angular velocity of the large rigid inertial component is $3.622 \times 10^{-4}$.

Figures 8 and 9 show that NISMC drives the attitude tracking errors to the origin faster than the existing FSMC and PD algorithms. In specific, NISMC takes $\sim 60 \mathrm{~s}$ for the error quaternion to converge to the equilibrium position, whereas the FSMC and PD require $\sim 80 \mathrm{~s}$ and $\sim 110 \mathrm{~s}$, respectively. Furthermore, NISMC takes $\sim 55 \mathrm{~s}$ for the error angular velocity to converge to the equilibrium position, whereas the FSMC and PD algorithms require $\sim 120 \mathrm{~s}$. In the steady-state response region, the final stable error quaternion and the final error of attitude angular velocity of the platform are $3.560 \times 10^{-7}$ and $1.798 \times 10^{-5}$, respectively, under the NISMC algorithm.

Figures 10 and 11 indicate that the NISMC takes $\sim 70 \mathrm{~s}$ for the error quaternion of the flexible solar panels to converge to the equilibrium position, whereas the FSMC and PD require $\sim 100 \mathrm{~s}$ and $\sim 110 \mathrm{~s}$, respectively. Furthermore, NISMC takes $\sim 75 \mathrm{~s}$ for the error of angular velocity to converge to the equilibrium position, whereas the FSMC and PD algorithms require $\sim 105 \mathrm{~s}$. The final stable error quaternion of the flexible solar panels is $1.704 \times 10^{-5}$ and the final error of attitude angular velocity of the platform is $1.114 \times 10^{-5}$, under the NISMC algorithm.

Table 1 presents the final stable error quaternion and the final error of attitude angular velocity of the platform under the FSMC and PD algorithms. Clearly, the magnitude of the error of angular velocity and error quaternion is smaller under the NISMC algorithm than under other control laws, indicating that the system has high steady-state accuracy.

These results demonstrate the superiority of the proposed NISMC algorithm in terms of faster convergence and higher stable accuracy over other control algorithms. 


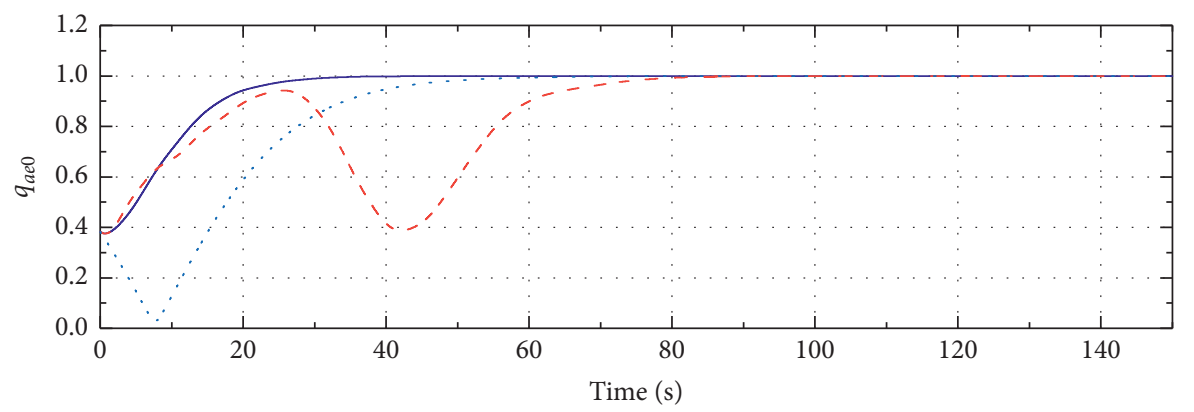

- NISMC

-.- FSMC

PD

(a)

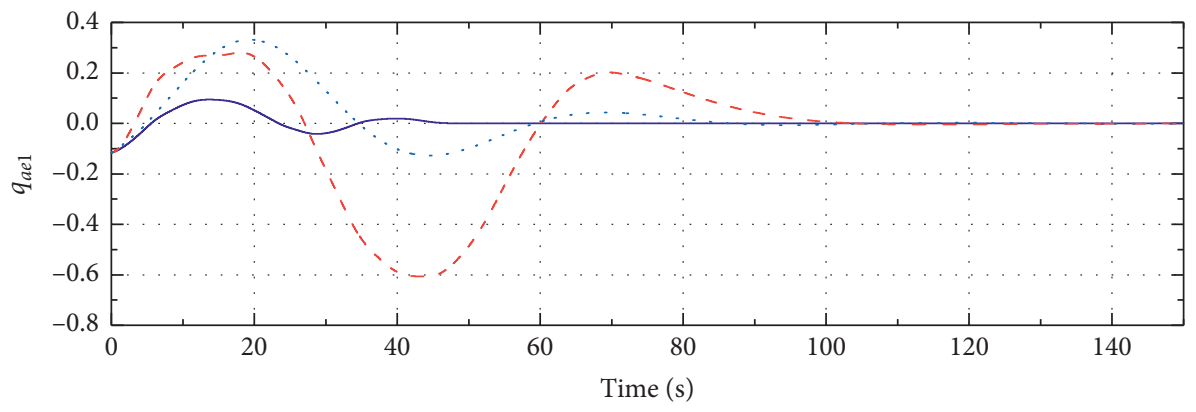

— NISMC

--- FSMC

..... $\mathrm{PD}$

(b)

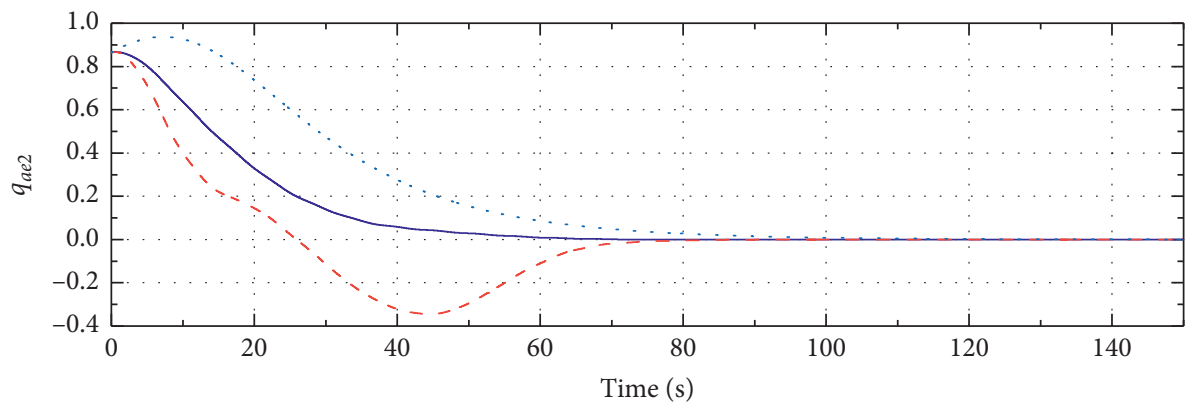

- NISMC

-. FSMC

PD

(c)

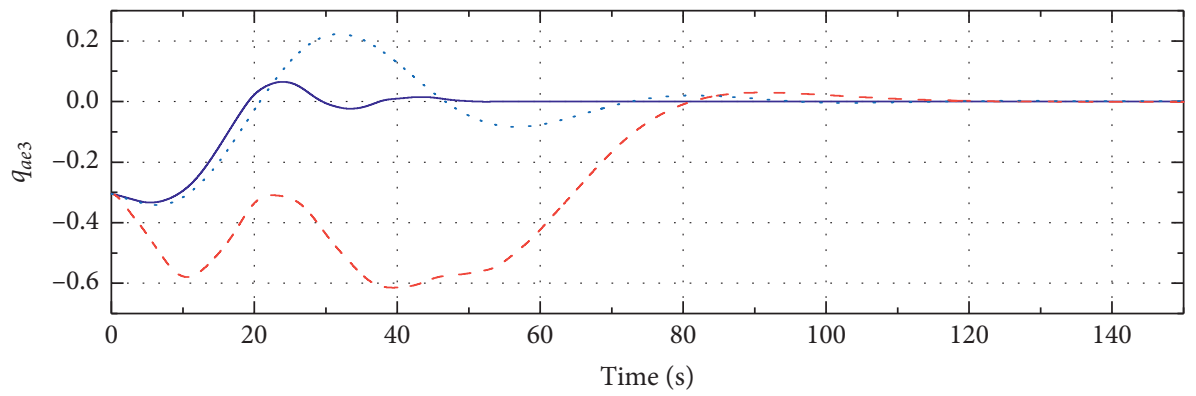

- NISMC

-. - FSMC

PD

(d)

Figure 10: Comparison of error quaternions of flexible solar panels. 


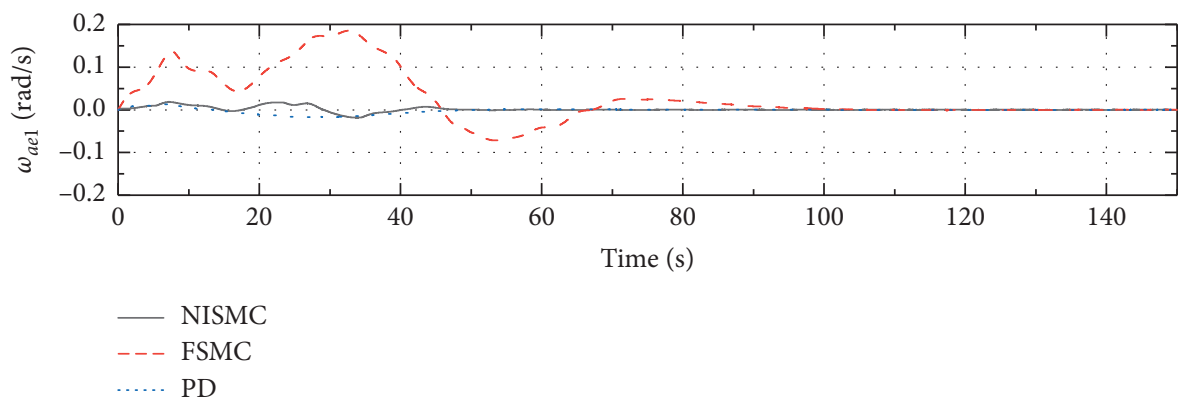

(a)

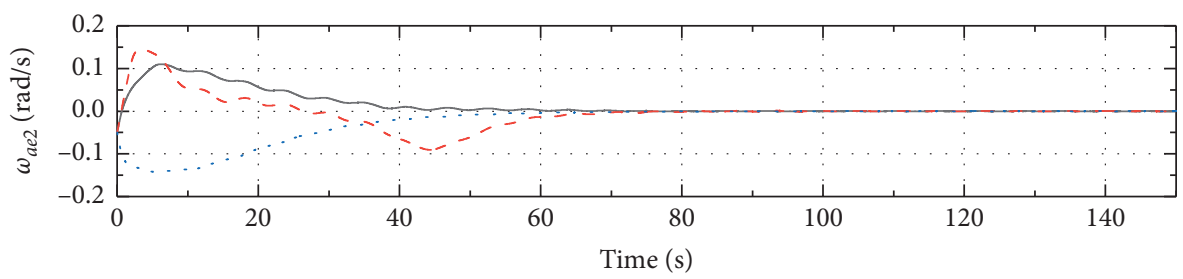

$\begin{array}{ll} & \text { NISMC } \\ --- & \text { FSMC } \\ \ldots \ldots & \text { PD }\end{array}$

(b)

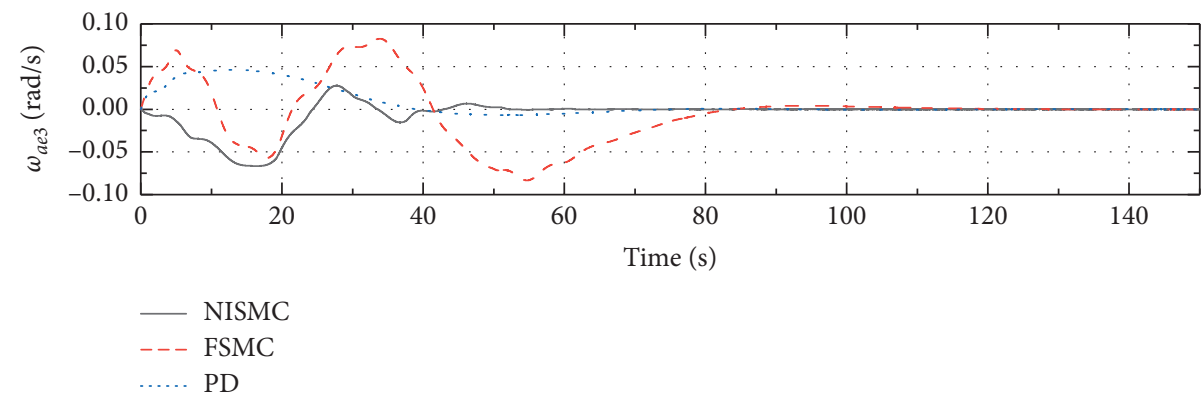

(c)

Figure 11: Comparison of errors of attitude angular velocity of flexible solar panels.

TABle 1: Parameter control performance.

\begin{tabular}{|c|c|c|c|}
\hline Control strategy & NISMC & FSMC & $\mathrm{PD}$ \\
\hline Stable error of attitude angular velocity of platform & $1.798 \times 10^{-5}$ & $5.074 \times 10^{-4}$ & 0.0012 \\
\hline Stable error of attitude angular velocity of flexible solar panels & $1.114 \times 10^{-5}$ & $1.243 \times 10^{-4}$ & 0.0018 \\
\hline Stable error quaternion of platform & $3.560 \times 10^{-7}$ & $2.574 \times 10^{-4}$ & 0.00391 \\
\hline Stable error quaternion of flexible solar panels & $1.704 \times 10^{-5}$ & $6.819 \times 10^{-4}$ & 0.00115 \\
\hline
\end{tabular}

\section{Conclusions}

A novel integral sliding mode control law was proposed for spacecraft systems with high-inertia components and flexible solar panels. The proposed control scheme exhibited faster convergence and provided precise attitude tracking in terms of external imbalances caused by appendages, rotation and vibration of flexible solar panels, and parametric uncertainties. Using numerical simulations, the control method was shown to allow an accurate tracking of the desired spacecraft attitude and the rotation of the flexible solar panels and high-inertia components around the special axis with a stable angular speed. Therefore, the control scheme offers a valuable contribution to the design of future space missions in both theoretical and practical respects. As future work, high-flexibility appendages, such as flexible antennas and connecting adapters as magnetic bearings, would be considered. Problems such as parameter uncertainty and actuator saturation demand advanced control methods to realize special state-constrained attitude maneuvering without any intrusion into the forbidden zones. Given the increasing complexity of space missions and the resulting changes to spacecraft design, I believe that this study will be of interest to those who work in aerospace engineering and similar fields. 


\section{Appendix}

The initial parameters of numerical simulation are as follows. The initial position of the spacecraft is $\mathbf{X}_{0}=\left[\begin{array}{lll}1097.540456 & 6040.616206 & -12.924317\end{array}\right]^{T} \mathrm{~km}$, and its initial velocity is $\mathbf{V}_{0}=\left[\begin{array}{lll}-7.450822 & 0.308646 & 1.538879\end{array}\right]^{T} \mathrm{~km} /$ $\mathrm{s}$ in the coordinate system $\mathrm{R}$. The initial quaternion of the platform is $\mathbf{Q}_{s}=\left[\begin{array}{llll}-0.3696 & -0.5301 & 0.6621 & -0.4372\end{array}\right]^{T}$, and its initial angular velocity is $\omega_{s 0}=\left[\begin{array}{lll}-0.02 & 0.015 & 0.025\end{array}\right]^{T}$. The initial angular velocity of the flexible solar panels is $\omega_{a 0}=\left[\begin{array}{lll}0 & 0 & 0\end{array}\right]_{T}^{T}$, and that of the high-inertia component is $\omega_{p 0}=\left[\begin{array}{lll}0 & 0 & 0\end{array}\right]^{T}$. The mass of the spacecraft platform is $1850 \mathrm{~kg}$. $J_{s 0}$ represents the inertia matrix of the spacecraft platform and is defined as

$$
J_{s 0}=\left[\begin{array}{ccc}
4938.75 & 0 & 0 \\
0 & 4642.63 & 0 \\
0 & 0 & 6607.31
\end{array}\right]
$$

The mass of a single flexible solar panel is $65 \mathrm{~kg}$. $J_{s a}$ represents the inertia matrix relative to a single flexible solar panel and is defined as

$$
J_{s a}=\left[\begin{array}{ccc}
251.385 & 35.56 & 1.417 \\
35.56 & 44.17 & -1.834 \\
1.417 & -1.834 & 875.97
\end{array}\right] \text {. }
$$

The mass of the high-inertia component is $800 \mathrm{~kg} . J_{s p}$ represents the inertia matrix relative to the high-inertia component and is defined as

$$
J_{s p}=\left[\begin{array}{ccc}
764.385 & 76.201 & 1.417 \\
76.201 & 652.17 & -2.17 \\
1.417 & -2.17 & 875.97
\end{array}\right] \text {. }
$$

The constant coefficient matrix of the vibration of solar panels relative to its rotation is

$$
F_{a}=\left[\begin{array}{cccccc}
-1.989 e 1 & -3.141 e-10 & -1.578 e-2 & 2.984 & -1.0287 & 3.945 e-3 \\
1.398 e-2 & 8.598 e-8 & -3.214 & 5.028 e-2 & 5.982 e-2 & 1.174 \\
2.791 e-8 & -1.913 & -5.027 e-8 & 3.13 e-10 & 2.016 e-9 & -5.279 e-9
\end{array}\right]
$$

The damping coefficient of the solar panel is 0.004 , and the modal frequency of the flexible solar panel is $\Omega_{a}=$ diag $[0.7033,0.8021,1.102,1.3006,2.755,3.044]$. Furthermore, $\Lambda_{a}=\Omega_{a}^{2}$. The coupling coefficient matrix that relates to the angle of the $+y$-axis between solar panels' vibration and platform's rotation is $f_{s a_{-} \text {right }}$, given by

$$
\begin{aligned}
f_{\text {sa_right_ } 1}= & {\left[\begin{array}{ccc}
1.996 e-10 & -1.425 e 1 & 8.016 e-13 \\
-2.451 e-2 & -2.016 & 5.946 e-3 \\
14.5 & 1.916 e-9 & 6.824 e-11
\end{array}\right], } \\
f_{\text {sa_right_2 }}= & {\left[\begin{array}{ccc}
-15.0146 & -2.125 e-9 & 2.616 e-9 \\
-2.451 & 2.516 e-2 & 4.346 e-9 \\
2.143 e-9 & 16.16 & 2.124 e-6
\end{array}\right], } \\
f_{\text {sa_right_3 }}= & {\left[\begin{array}{ccc}
-2.842 e-8 & 8.164 e-3 & -1.921 e-10 \\
1.274 e-4 & 9.524 e-3 & -2.014 \\
-8.302 e-3 & -2.840 e-8 & -1.56 e-8
\end{array}\right], } \\
f_{\text {sa_right_4 }}= & {\left[\begin{array}{ccc}
-1.519 e-9 & 3.441 & 2.502 e-11 \\
1.376 e-2 & -0.546 & -3.014 e-2 \\
-3.412 & -5.840 e-9 & 2.021 e-8
\end{array}\right], } \\
f_{\text {sa_right_5 }}= & {\left[\begin{array}{ccc}
5.842 e-9 & -1.245 & -1.721 e-10 \\
-1.204 e-3 & -0.629 & 2.514 e-2 \\
1.425 & -6.840 e-10 & -1.462 e-8
\end{array}\right], } \\
f_{\text {sa_right_6 }}= & {\left[\begin{array}{ccc}
-1.627 e-9 & -5.314 e-2 & -2.701 e-10 \\
2.204 e-5 & 2.429 e-2 & 1.017 \\
-0.7253 & -1.747 e-8 & -1.629 e-10
\end{array}\right] . }
\end{aligned}
$$

The constant matrix of the $-y$-axis solar panels' vibration to the platform's rotation is $f_{s a_{-} \text {left }}$ and is defined as

$$
\begin{aligned}
& f_{s a_{-} \text {left_1 }}=\left[\begin{array}{ccc}
1.923 e-10 & -1.464 e 1 & 1.107 e-12 \\
-2.397 e-2 & -2.111 & 5.589 e-3 \\
14.745 & 1.867 e-9 & 6.977 e-11
\end{array}\right], \\
& f_{\text {sa_left_2 }}=\left[\begin{array}{ccc}
-15.7046 & -2.1074 e-9 & 2.819 e-9 \\
-2.5415 & 2.624 e-2 & 4.402 e-9 \\
2.271 e-9 & 1.5963 e 1 & 9.924 e-5
\end{array}\right] \text {, } \\
& f_{\text {sa_left_3 }}=\left[\begin{array}{ccc}
-2.822 e-8 & 8.132 e-3 & -1.893 e-10 \\
1.24 e-4 & 9.245 e-3 & -1.9184 \\
-8.295 e-3 & -2.789 e-8 & -1.506 e-8
\end{array}\right] \text {, } \\
& f_{s a_{-} \text {left_}_{4}}=\left[\begin{array}{ccc}
-1.589 e-9 & 3.941 & 2.752 e-11 \\
1.731 e-2 & -0.740 & -3.214 e-2 \\
-3.410 & -5.836 e-9 & 2.122 e-8
\end{array}\right] \text {, } \\
& f_{s a_{-} \text {left_5 }}=\left[\begin{array}{ccc}
5.857 e-9 & -1.347 & -1.691 e-10 \\
-1.344 e-3 & -0.867 & 2.474 e-2 \\
1.476 & -6.753 e-10 & -1.512 e-8
\end{array}\right] \text {, } \\
& f_{s a_{-} \text {left_6 }}=\left[\begin{array}{ccc}
-1.616 e-9 & -5.324 e-2 & -2.671 e-10 \\
2.134 e-5 & 2.527 e-2 & 1.457 \\
-0.923 & -1.547 e-8 & -1.591 e-10
\end{array}\right] \text {. }
\end{aligned}
$$


Here, $r_{\text {sa_right }}$ represents the coupling rotation coefficient matrix of the $+y$-axis between the solar panels and the platform; similarly, $r_{s a_{-} \text {left }}$ is given as

$$
\begin{aligned}
& r_{s a_{-} \text {right_1 }}=\left[\begin{array}{ccc}
5.996 e-11 & 0 & 6.213 e-3 \\
0 & 4.88 e 1 & 1.613 e-12 \\
-3.75 e 2 & 0 & 1.216 e-6
\end{array}\right], \\
& r_{s a_{-} \text {right_2 }}=\left[\begin{array}{ccc}
-1.329 e-7 & 0 & -1.717 e-1 \\
0 & -8.964 e-5 & 0 \\
1.826 e-3 & 1.023 e-13 & 1.071 e-10
\end{array}\right], \\
& r_{s a_{-} \text {right_3 }}= {\left[\begin{array}{ccc}
3.462 e 2 & 0 & -1.213 e-6 \\
0 & 1.0121 & 0 \\
-4.956 e-9 & 0 & 9.016 e-3
\end{array}\right], } \\
& f_{s a_{-} \text {left_1 }}= {\left[\begin{array}{ccc}
6.106 e-11 & 0 & 6.115 e-3 \\
0 & 4.69 e 1 & 1.718 e-12 \\
-3.68 e 2 & 0 & -1.203 e-6
\end{array}\right], } \\
& f_{s a_{-} \text {left_3 }}=\left[\begin{array}{ccc}
3.501 e 2 & 0 & -1.116 e-6 \\
0 & 1.0121 & 0 \\
-4.755 e-9 & 0 & 9.314 e-3
\end{array}\right], \\
& f_{s a_{-} \text {left_2 }}=\left[\begin{array}{ccc}
-1.006 e-7 & 0 & -1.998 e-1 \\
0 & -8.964 e-5 & 0 \\
1.904 e-3 & -1.021 e-13 & 1.176 e-10
\end{array}\right],
\end{aligned}
$$

Furthermore, $r_{s p}$ represents the constant rotating coupling coefficient matrix between the high-inertia component and the platform and is given as

$$
\begin{aligned}
r_{s p_{-} 1} & =\left[\begin{array}{ccc}
2.206 e-6 & -1.128 e-6 & 6.213 e-3 \\
0 & 4.88 e-8 & 0 \\
-3.75 e-12 & 0 & 1.216 e-8
\end{array}\right], \\
r_{s p_{-} 2} & =\left[\begin{array}{ccc}
3.173 e-5 & 0 & -1.213 e-7 \\
0 & 1.012 e-7 & 1.038 e-9 \\
-1.097 e-12 & 0 & 9.016 e-11
\end{array}\right], \\
r_{s p_{-} 3} & =\left[\begin{array}{ccc}
3.462 e-5 & 0 & -1.213 e-6 \\
2.277 e-7 & 5.02 e-8 & 0 \\
-4.956 e-10 & 0 & 4.414 e-13
\end{array}\right] .
\end{aligned}
$$

\section{Data Availability}

The data used to support the findings of this study are included within the article.

\section{Conflicts of Interest}

The authors declare that there are no conflicts of interest regarding the publication of this paper.

\section{Acknowledgments}

This work was supported by the National Natural Science Foundation of China (nos. 61833009, 61690212, and 91438202) and the National Key Research and Development Plan (no. 2016YFB0500901).

\section{References}

[1] C. Sendi and M. A. Ayoubi, "Robust fuzzy tracking control of flexible spacecraft via a T-S fuzzy model," IEEE Transactions on Aerospace and Electronic Systems, vol. 54, no. 1, pp. 170-179, 2017.

[2] Z. Liu, J. Liu, and L. Wang, "Disturbance observer based attitude control for flexible spacecraft with input magnitude and rate constraints," Aerospace Science and Technology, vol. 72, pp. 486-492, 2018.

[3] Z. Li, L. Gao, W. Chen, and Y. Xu, "Distributed adaptive cooperative tracking of uncertain nonlinear fractional-order multi-agent systems," IEEE/CAA Journal of Automatica Sinica, vol. 7, no. 1, pp. 292-300, 2020.

[4] Q. Zhou, G. Chen, R. Lu, and W. Bai, "Disturbance-observerbased event-triggered control for multi-agent systems with input saturation," Scientia Sinica Informationis, vol. 49, no. 11, pp. 1502-1516, 2019.

[5] Y. Li, K. Li, and S. Tong, "Finite-time adaptive fuzzy output feedback dynamic surface control for MIMO nonstrict feedback systems," IEEE Transactions on Fuzzy Systems, vol. 27, no. 1, pp. 96-110, 2019.

[6] S. Tong, X. Min, and Y. Li, "Observer-based adaptive fuzzy tracking control for strict-feedback nonlinear systems with unknown control gain functions," IEEE Transactions on Cybernetics, vol. 50, no. 9, pp. 3903-3913, 2020.

[7] L. Chen, P. Shi, and M. Liu, "Fault reconstruction for Markovian jump systems with iterative adaptive observer," Automatica, vol. 105, pp. 254-263, 2019.

[8] L. Zhao, J. Yu, and P. Shi, "Command filtered backsteppingbased attitude containment control for spacecraft formation," IEEE Transactions on Systems, Man, and Cybernetics: Systems, vol. 51, no. 2, p. 1278, 2021.

[9] Z. Yang, N. Lu, J. Shi, P. Zhang, C. Dong, and J. Yang, "Overview of FY-3 payload and ground application system," IEEE Transactions on Geoscience and Remote Sensing, vol. 50, no. 12, pp. 4846-4853, 2012.

[10] J. Han, The Technique for Estimating and Compensating the Uncertainties: Active Disturbance Rejection Control Technique, National Defense Industry Press, Beijing, China, 2008.

[11] X. Cao, P. Shi, Z. Li, and M. Liu, "Neural-network-based adaptive backstepping control with application to spacecraft attitude regulation," IEEE Transactions on Neural Networks and Learning Systems, vol. 29, no. 9, pp. 4303-4313, 2018.

[12] Q. Liu, M. Liu, and J. Yu, "Adaptive fault-tolerant control for attitude tracking of flexible spacecraft with limited data transmission," IEEE Transactions on Systems, Man, and Cybernetics: Systems, p. 1, 2019.

[13] M. Liu, L. Zhang, P. Shi, and Y. Zhao, "Fault estimation sliding-mode observer with digital communication constraints," IEEE Transactions on Automatic Control, vol. 63, no. 10, pp. 3434-3441, 2018.

[14] S. Yu, X. Yu, B. Shirinzadeh, and Z. Man, "Continuous finite-time control for robotic manipulators with terminal sliding mode," Automatica, vol. 41, no. 11, pp. 1957-1964, 2005.

[15] A. Sofyalı and E. Jafarov, "Robust stabilization of spacecraft attitude motion under magnetic control through time varying 
integral sliding mode," International Journal of Robust and Nonlinear Control, vol. 29, no. 11, pp. 3446-3468, 2019.

[16] Q. Li, L. Liu, Y. Deng, S. Tang, and Y. Zhao, "Twistor-based synchronous sliding mode control of spacecraft attitude and position," Chinese Journal of Aeronautics, vol. 31, no. 5, pp. 1153-1164, 2018.

[17] W. Liu, Z. Feng, and A. Bi, "A novel nonsingular terminal sliding mode control combined with global sliding surface for uncertain nonlinear second-order systems," Transactions of the Institute of Measurement and Control, vol. 42, no. 7, pp. 1294-1300, 2020.

[18] L. Zhao, G. Liu, and J. Yu, "Finite-time adaptive fuzzy tracking control for a class of nonlinear systems with fullstate constraints," IEEE Transactions on Fuzzy Systems, p. 1, 2020.

[19] K. Lu, Y. Xia, Z. Zhu, and M. V. Basin, "Sliding mode attitude tracking of rigid spacecraft with disturbances," Journal of the Franklin Institute, vol. 349, no. 2, pp. 413-440, 2012.

[20] A. Yousefpour and H. Jahanshahi, "Fast disturbance-observerbased robust integral terminal sliding mode control of a hyperchaotic memristor oscillator," The European Physical Journal Special Topics, vol. 228, no. 10, pp. 2247-2268, 2019.

[21] S. Lv, N. Wang, X. Liang, and M. Er, "Finite-time disturbance observer based nonsingular integral terminal sliding mode trajectory tracking control of unmanned surface vehicles," in Proceedings of the 2016 IEEE Chinese Guidance, Navigation and Control Conference (CGNCC), pp. 1109-1113, Nanjing, China, 2016.

[22] J. Na, "Adaptive prescribed performance control of nonlinear systems with unknown dead zone," International Journal of Adaptive Control and Signal Processing, vol. 27, no. 5, pp. 426-446, 2013.

[23] Q. Chen, X. Ren, J. Na, and D. Zheng, “Adaptive robust finite-time neural control of uncertain PMSM servo system with nonlinear dead zone," Neural Computing and Applications, vol. 28, no. 12, pp. 3725-3736, 2016.

[24] N. Shi, Z. Kang, Z. Zhao, and Q. Meng, "Adaptive vector nonsingular terminal sliding mode control for a class of n-order nonlinear dynamical systems with uncertainty," Mathematical Problems in Engineering, vol. 2020, Article ID 7309417, 12 pages, 2020.

[25] C. Yue, K. D. Kumar, Q. Shen, C. H. Goh, and T. H. Lee, "Attitude stabilization using two parallel single-gimbal control moment gyroscopes," Journal of Guidance, Control, and Dynamics, vol. 42, no. 2, pp. 1353-1364, 2019.

[26] S. Alshamali and E. Aljuwaiser, "Design of robust observerbased backstepping control for a satellite control system," Mathematical Problems in Engineering, vol. 2019, Article ID 7412194, 9 pages, 2019.

[27] J. Wang, L. Liu, C. Liu, and X. Li, “Adaptive sliding mode control based on equivalence principle and its application to chaos control in a seven-dimensional power system," Mathematical Problems in Engineering, vol. 2020, Article ID 1565460, 13 pages, 2020.

[28] P. Li, J. Ma, Z. Zheng, and L. Geng, "Fast nonsingular integral terminal sliding mode control for nonlinear dynamical systems," in Proceedings of the 2014 IEEE 53rd Annual Conference On Decision And Control (CDC), pp. 4739-4746, IEEE, Los Angeles, CA, USA, 2015.

[29] Y. Miao, I. Hwang, M. Liu, and F. Wang, "Adaptive fast nonsingular terminal sliding mode control for attitude tracking of flexible spacecraft with rotating appendage," Aerospace Science and Technology, vol. 93, Article ID 105312, 2019.
[30] H. Gui and G. Vukovich, "Adaptive fault-tolerant spacecraft attitude control using a novel integral terminal sliding mode," International Journal of Robust and Nonlinear Control, vol. 27, no. 16, pp. 3174-3196, 2017.

[31] Y. Xie, Research on Dynamic Balance Technique of Satellite with Large Rotating Components, Harbin Institute of Technology, Harbin, China, 2019.

[32] L. Chai, On-orbit Estimation of Unbalanced Torques Acting on Satellite Platform with Large Inertia Rotating Payloads [D], " Harbin Institute of Technology, Harbin, China, 2019.

[33] Z. Zhu, Y. Xia, and M. Fu, "Attitude stabilization of rigid spacecraft with finite-time convergence," International Journal of Robust and Nonlinear Control, vol. 21, no. 6, pp. 686-702, 2011.

[34] Q. Hu, X. Huo, and B. Xiao, "Reaction wheel fault tolerant control for spacecraft attitude stabilization with finite-time convergence," International Journal of Robust Nonlinear Control, vol. 23, no. 8, pp. 1737-1752, 2012.

[35] G. Hardy, J. Littlewood, and G. Pólya, Inequalities, Cambridge University Press, Cambridge, UK, 1952.

[36] S. P. Bhat and D. S. Bernstein, "Finite-time stability of continuous autonomous systems," SIAM Journal on Control and Optimization, vol. 38, no. 3, pp. 751-766, 2000.

[37] C. Zhong, L. Wu, J. Guo, Y. Guo, and Z. Chen, "Robust adaptive attitude manoeuvre control with finite-time convergence for a flexible spacecraft," Transactions of the Institute of Measurement and Control, vol. 40, no. 2, p. 425, 2016.

[38] L. Yang and J. Yang, "Nonsingular fast terminal sliding-mode control for nonlinear dynamical systems," International Journal of Robust and Nonlinear Control, vol. 21, pp. 18651879, 2011. 